\title{
Investigation of Model Reduction Using Bayesian Calibration and Dynamic Discrepancy
}

\author{
Ahmed Nirjhar Alam
}

Follow this and additional works at: https://researchrepository.wvu.edu/etd

\section{Recommended Citation}

Alam, Ahmed Nirjhar, "Investigation of Model Reduction Using Bayesian Calibration and Dynamic Discrepancy" (2018). Graduate Theses, Dissertations, and Problem Reports. 7151.

https://researchrepository.wvu.edu/etd/7151

This Thesis is protected by copyright and/or related rights. It has been brought to you by the The Research Repository @ WVU with permission from the rights-holder(s). You are free to use this Thesis in any way that is permitted by the copyright and related rights legislation that applies to your use. For other uses you must obtain permission from the rights-holder(s) directly, unless additional rights are indicated by a Creative Commons license in the record and/ or on the work itself. This Thesis has been accepted for inclusion in WVU Graduate Theses, Dissertations, and Problem Reports collection by an authorized administrator of The Research Repository @ WVU. For more information, please contact researchrepository@mail.wvu.edu. 


\title{
Investigation of Model Reduction Using Bayesian Calibration and Dynamic Discrepancy
}

\begin{abstract}
Ahmed Nirjhar Alam
Thesis submitted to the Benjamin M. Statler College of Engineering and Mineral Resources at West Virginia University in partial fulfillment of the requirements for the degree of Master of Science in Mechanical Engineering
\end{abstract}

\author{
David S. Mebane, Ph.D., Chair \\ Fernando V. Lima, Ph.D. \\ Konstantinos Sierros, Ph.D. \\ Department of Mechanical and Aerospace Engineering \\ Morgantown, West Virginia \\ 2018
}

Keywords: Model Reduction, Dynamic Discrepancy, Uncertainty Quantification, Bayesian Calibration, Markov Chain Monte Carlo, Steam Methane Reformation

Copyright 2018 Ahmed Nirjhar Alam 


\title{
ABSTRACT
}

\section{Investigation of Model Reduction Using Bayesian Calibration and Dynamic Discrepancy}

\author{
Ahmed Nirjhar Alam
}

Rapid and consistent increase in computational power availability has given rise to more and more implementation of computational models to simulate complex processes described by mathematical models. With this trend, reducing the computational cost of a model is of significant importance now. This work explores a technique of reducing a model by quantifying the model discrepancy and subsequently uses the reduced model for making predictions. The final predictions show satisfactory agreement with the actual data or 'truth'.

All models make simplifying assumptions. This deviates the model from the actual physical process. Also, many natural processes contain random factors that can not be modeled efficiently. Together these two factors lead to disparity between the model and the actual process. This difference is called model inadequacy or model discrepancy. Significant amount of work has been done so far in quantifying model discrepancy. These terms quantifying model discrepancy can be included in the model equations as corrections. Various techniques for performing these corrections have been developed in past literature.

Alternatively, the same method for quantifying model discrepancy can be used to reduce a computationally intractable model. This would involve reducing the larger high fidelity model to a simpler form. These reductions would therefore be the equivalent to the simplifying assumptions discussed before. Of course this trimming of the model would have to be done judiciously to ensure that the core behavior of the model remains unchanged. Reducing the model in this manner will doubtlessly introduce new sources of model discrepancy. The discrepancy introduced from this reduction process can then be modeled using stochastic functions. In effect, this will simply reduce the number of Ordinary Differential Equations(ODEs) describing the dynamic system and substitute the information that was contained in them with inexpensive basis functions modeling discrepancy. This will result in a significant decrease in computational cost, which is the objective of this work.

To conduct this study, steam methane reformation(SMR) reaction, occurring in Continuously Stirred Tank Reactor(CSTR), was used as an example case. A reduced model of the Steam Methane Reformation Reaction was built using the reaction network from the pioneering work of $\mathrm{Xu}$ and Froment. This model was then calibrated to the experimental data found in the paper using the process of Bayesian Calibration with Markov Chain Monte Carlo (MCMC) sampling. The calibration produced a reasonable posterior distribution of the physical parameters for the reaction, which 
are of scientific interest, and will exhibit a similar distribution for reasonable cases of extrapolation of process conditions. Although an initial calibration of the physical parameters only produced a reasonable distribution for some of the parameters, the coverage obtained with model prediction in this initial phase was unsatisfactory. This is to be expected, since the loss of details in the reduced order model would doubtlessly affect model prediction. After calibrating the physical parameters, discrepancy terms were added incrementally to the reduced model, each accounting for specific interactions that the reduced model did not consider. After each addition, the model was re-calibrated to the data and the model prediction coverage was observed. The addition of the discrepancy terms achieved significant improvement of the model predictions and enabled them to cover the data with a reasonable amount of uncertainty. The final reduced model also displayed the capability to replicate experimental data for interpolation cases. Subsequently, the final reduced model was extended to industrial scale, represented by a plug flow reactor. 


\section{Contents}

1 Introduction 1

1.1 Short Overview of Model Reduction Techniques and Model Discrepancy Quantification ................... . . 1

1.2 Steam Methane Reformation Reaction . . . . . . . . . . . . . 5

2 Model Development $\quad 12$

2.1 Model Reduction . . . . . . . . . . . . . . . . . . . . . 12

2.2 Model Kinetics . . . . . . . . . . . . . . . . . . . . . . . . . . 16

2.3 Model Solver . . . . . . . . . . . . . . . . . . . . . . . 20

2.4 Discrepancy Function . . . . . . . . . . . . . . . . . . . . . 21

2.5 Bayesian Calibration . . . . . . . . . . . . . . . 25

2.6 Calibration Data . . . . . . . . . . . . . . . . 29

2.6.1 CSTR Assumption . . . . . . . . . . . . . . . 30

3 Calibration and Results 31

3.1 Calibration Plots . . . . . . . . . . . . . . . . . . . 32

3.2 Plug Flow Reactor(PFR) . . . . . . . . . . . . . . . . . . 43

3.3 Discussions and Future Work . . . . . . . . . . . . . . . . 45

4 Acknowledgements $\quad 46$

$\begin{array}{lll}5 & \text { Citations } & 47\end{array}$

\section{List of Figures}

1 Xu-Froment Proposed Reaction Scheme[15] . . . . . . . . . . . . . . . 9

$2 \quad$ Idealized Full Reaction Network[38] . . . . . . . . . . . . . . . . . . . 13

$3 \quad$ Reduced Reaction Network[38] . . . . . . . . . . . . . . . . . . . . 13

$4 \quad \mathrm{Xu}$ and Froment Full Reaction Network[38] . . . . . . . . . . . . . . . 14

$5 \quad \mathrm{Xu}$ and Froment Reduced Reaction Network[38] . . . . . . . . . . . . 15

$6 \quad \operatorname{CSTR}[39] \ldots \ldots \ldots \ldots \ldots \ldots$

$7 \quad$ Basis Functions of Different Orders[42] . . . . . . . . . . . . . . . 24

8 Example Calibration Data[15] . . . . . . . . . . . . . . . 29

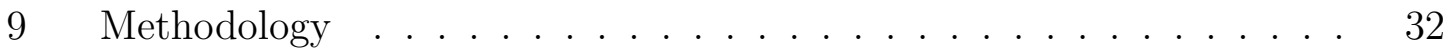

10 Scatter Plot - Physical Parameters . . . . . . . . . . . . . . 33

11 Scatter Plots for Calibration without Discrepancy . . . . . . . . . . . 34

12 Parameter Estimation Plots without Discrepancy I . . . . . . . . . . 35

13 Parameter Estimation Plots without Discrepancy II . . . . . . . . . . 36

14 Model Prediction without Discrepancy . . . . . . . . . . . . . . 37

15 Model Prediction With Single Beta Discrepancy Function . . . . . . . 38

16 Distribution of Sample Beta Parameters for Main Effects . . . . . . . 39

17 Beta Parameters Estimation Plots for Main Effects Calibration . . . . 40

18 Prediction for Model With All Main Effects Incorporated . . . . . . . 40 
19 BIC for Different Models . . . . . . . . . . . . . . . . . . . . . . . . . 41

20 Optimized Model Prediction . . . . . . . . . . . . . . . . . . 42

21 Plug Flow Reactor[44] . . . . . . . . . . . . . . . . . . . 43

22 PFR Output With Optimum Model . . . . . . . . . . . . . . . . . . . 44

\section{List of Tables}

1 Discrepancy Function Inputs for $\kappa_{1} \ldots \ldots \ldots 22$

2 Parameter Bounds . . . . . . . . . . . . . . . . . . . 27

3 Parameter Bounds With Log-scale Sampling . . . . . . . . . . . . . . 27 


\section{Introduction}

The increasing complexity of computer models used to simulate real world processes means that model reduction is a desired attribute in most scientific endeavors. This importance is further augmented with the recent popularity of learning algorithms that require large numbers of model runs to acquire hidden intrinsic patterns to the underlying process[1]. This work discusses a technique of model reduction that takes into account the model discrepancy. Model discrepancy, in the context of this work, refers to inadequacy of the model. It is the discrepancy between the actual physical process and the model that is introduced due to the layers of details not taken into account by the model, usually in the form of simplifying assumptions made about the actual physical process. Also, most natural processes contain certain random elements that cannot be modeled analytically. Together, these factors lead to certain disagreement between the model results and the actual process data, even when the model is at its most accurate. This is referred to as model discrepancy or model inadequacy. This work attempts to quantify this model discrepancy for a certain range of input values and subsequently uses the discrepancy to satisfactorily make accurate predictions with the reduced model.

\subsection{Short Overview of Model Reduction Techniques and Model Discrepancy Quantification}

The use of model reduction techniques in both scientific and engineering endeavours is quite wide-spread. In chemical kinetics, quasi steady-state assumption techniques are frequently used for model reduction. These methods assume that certain intermediates in a reaction reach steady state conditions in a shorter period of time. The assumption of steady state enables these intermediate species to be related by a simple algebraic equation consisting of equilibrium constants rather than elaborate and expensive differential equations, hence driving down the computational cost of the model[2]. Another extensively applied method of model reduction is the lumped species method[3]. This involves lumping of several species into a single pseudospecies, hence reducing the number of states in the model. The species chosen for this will depend on the dynamic features of the specific model in consideration. Sensitivity analysis techniques involve identification of states and parameters that have little contribution on the response of the system at a particular configuration[4]. These components are then eliminated or their impact is reduced to a skeletal form. Time-scale analysis identifies the different scales over which species react. The fast time-scale reactions and species are assumed to be at steady state and are solved using their equilibrium constants. The slower reactions are taken into account in the form of differential equations. Proper Orthogonal Decomposition(POD) aims to achieve a reduced order model by projecting the high-fidelity model onto a reduced space basis[5]. If the basis is chosen appropriately, the relevant high fidelity system dynamics can be captured with a greatly reduced number of states. The range of validity of the reduced-order model is determined by the specifics of the reduction procedure. 
Unlike the methods discussed, the use of dynamic discrepancy for model reduction involves quantification of the uncertainty associated with the reduced model. This is often accompanied with the identification of the different sources of uncertainty. This produces a predictive distribution for the reduced model instead of definite predictions. This reporting of the uncertainty associated with a particular model prediction makes this technique useful for scenarios where wrong or overconfident predictions can be catastrophic. Also, the reduction of a model by dynamic discrepancy can also be more generic than other conventional methods. It can be implemented without detailed knowledge of the process involved. Simply identifying the dependencies in the dynamic system may be enough in certain situations to reduce a model using dynamic discrepancy, as will be demonstrated in this work.

Kennedy and O'Hagan introduced the idea of model discrepancy and how disregarding it leads to biased and over-confident model predictions in their landmark publication in 2001 [6]. Their work categorized the different sources of uncertainty in computer models and subsequently demonstrated how explicitly modeling them leads to better prediction and uncertainty quantification. A multivariate joint Gaussian distribution was used to model the prior distribution of discrepancy, a concept that was extended to this work. Brynjarsdottir and O'Hagan extended on their work and investigated the predictive capabilities of the model for cases of extrapolation[7]. Their work found that in order to do reasonable extrapolations with a model containing discrepancy, simply modeling the discrepancy was not enough. The prior distribution chosen for the discrepancy function was also critical when it came to the fidelity of the extrapolation predictions. It was also noted that an overly wide prior, or a prior distribution positioned incorrectly, may also lead to model predictions that break physical laws or hold no meaning. Their work also connected the problem of model uncertainty quantification with discrepancy. Uncertainty quantification is a major issue with the almost ubiquitous computer models used these days, since it quantifies how accurate and reliable a given model is. However, when it comes to quantifying uncertainty, many models only take into account the uncertainty associated with the input variables used and propagate that to the model prediction. However, this is clearly only a partial quantification of the uncertainty since it disregards model inadequacy, which is also a major source of error.

Joseph and Melkote used quantification of model uncertainty and demonstrated the various facets involved with it using a problem of predicting surface roughness in a micro cutting process and another problem of optimization of a spot welding process[8].They demonstrated that purely statistical models, that is models built from observation data only without any understanding of the underlying physical process incorporated into it, only perform well within conditions from which the observation were obtained. Model predictions beyond this specific range of conditions have poor accuracy. On the other hand physical models, that is models that were built solely based on the intrinsic physical process without the augmentations of observation data, do not always match satisfactorily with reality. A combination of both, termed statistical-engineering models, combine the best of both models and mitigate their 
drawbacks, producing predictions with a higher fidelity compared to the other two types of models. The engineering-statistical model was built sequentially, with statistical adjustments made to the physical model at each step and the model inadequacy evaluated. When model inadequacy is at an acceptable value, the adjustments are stopped. This method of model building ensures that the physical model always play a part in the final model, thereby ensuring that the model output's basic structure is preserved. This approach was used in this work with the process of adding the discrepancy functions.

Sargsyan et al. presented a novel method for incorporating dynamic discrepancy terms in the physical model[9]. The discrepancy terms, in their work, were embedded in the values of the parameters instead of being added explicitly to equations for the quantities of interest. This, they argued, served the following purposes.

First, additions of model inadequacy terms explicitly to the equations for output variables is more likely to break various natural constraints associated with the physical process and its physical equations. An example in the context of this work could be the expression for partial pressure change for methane in a Continuously Stirred Tank Reactor (CSTR). The total pressure inside the tank is constant throughout the process. Suppose the change in pressure with time is represented by the equation below.

$$
\frac{d P_{C H_{4}}}{d t}=f(x, \lambda)+\epsilon_{m}(x)
$$

where $\mathrm{x}$ is vector of input variables and $\lambda$ is the vector of model parameters and $\epsilon_{m}(x)$ is the discrepancy function correcting for model inadequacy. Suppose, initially, methane occupies the whole tank. In that stage, if $\epsilon_{m}(x)$ is greater than $\mathrm{f}(\mathrm{x}, \lambda)$, $\frac{d P_{C H_{4}}}{d t}$ will be positive, leading methane to overflow the tank and break the physical constraint $P_{\text {total }}=$ constant. A bounded value for $\epsilon_{m}(x)$ may solve this issue, but finding such bounds for all possible scenarios is difficult. Also, an overly restrictive bound may prevent true values of $\epsilon_{m}(x)$ from being discovered during calibration.

Secondly, explicit addition of discrepancy terms to specific quantities of interest may only correct the bias for those specific quantities and therefore will produce poor results when we change the quantity of interest. $y=f(x, \lambda)+\epsilon(x)$ will not correct bias properly for $\mathrm{z}=\mathrm{g}(\mathrm{x})$, where $\mathrm{y}$ and $\mathrm{z}$ are two different quantities of interest.

Finally, the calibrated model $\mathrm{y}=f(x, \lambda)+\epsilon(x)$ is not the actual physical model but its alteration in a somewhat arbitrary fashion. It changes the core structure of the physical model and its output pattern. Given that a lot of time and thoughts are spent by experts on coming up with these models, obliterating the integral structure of these models with addition of explicit statistical terms is unlikely to be acceptable.

In their work, Sargsyan et al. demonstrate that embedding the model inadequacy internally in the expression for physical parameters alleviates these problems. It essentially updates the assumptions made in the physical model.

The majority of the work on modeling uncertainty in the past few years has focused on procedures for quantifying observational error but similar methods for model error/inadequacy has not evolved as much[7]. The work Sargsyan et al. contribute to 
the studies of model error/inadequacy by attempting to overcome the shortcomings of explicit model correction. They embed the model error terms in the parameter expressions and both existing model parameters and parameters that quantify model error are inferred simultaneously during calibration. This approach leads to a model error representation that is consistent with underlying physics and can also be used as a device to attribute model error to certain specific parts of the model. This eventually leads to better quantification of sources of uncertainty.

Mebane, Bhat et al. implemented a similarly intrusive form of model uncertainty quantification with discrepancy terms incorporated in the differential equations describing the system, resulting in stochastic differential equations[10]. Modeling the uncertainty in this manner enabled the up-scaling of uncertainty by propagation for both model parameters and discrepancy parameters to the large scale system. Similar methods of uncertainty quantification have been used in various past literatures $[11,12,13]$ and hence was relied upon to model discrepancy in this work.

Higdon et al. implemented a similar technique of quantifying model inadequacy and other sources of uncertainty on a spot welding process and a charged particle accelerator. He and Xiu, in one of their works with discrepancy, used both external and internal model corrections. They also referred to one of the broad problems of model correction with discrepancy, which is how it can lead to violation of the physical model's constraints. As a remedy, they came up with the framework PCMC, which stands for physics constrained model correction. Their work incorporates the physical constraints into the new statistically adjusted model and makes it one of the model parameters. Internal and external corrections are described as follows in the context of their work.

External model corrections were describes as follows in their work.

$$
M_{c}=M(x ; p(x))+\delta_{m}(x)
$$

where $\mathrm{p}(\mathrm{x})$ is an important model process, $\mathrm{x}$ is model input and $M_{c}$ is corrected model and $\delta_{m}(x)$ is the discrepancy term.

On the other hand, internal model correction involved the following adjustments

$$
\begin{gathered}
p_{c}(x)=p(x)+\delta_{m}(x) \\
M=M(x ; p(x))
\end{gathered}
$$

The internal model correction in the above equations is a more intrusive method that has the capacity to preserve the physical constraints of the model. It can also change the overall output of the model, ensuring all possible quantities of interest(QoI) are altered by taking into account model inadequacy. Oliver et al. propose and explore a validation and predictive assessment process that supports extrapolative predictions for models that has been corrected for known sources of error.[14] In the classical approaches of model building, after calibration, the model prediction is compared to a set of real data. If sufficient agreement is observed, the model is 
considered validated. However, validations of this kind are precarious because they only ensure that the model is accurate for specific range of conditions and for a specific QoI. They say little of the fidelity of the model for cases of extrapolation or for different QoIs. Their work established a generalized framework for validation of a model's extrapolative predictions that can help alleviate this problem.

For this work, the steam methane reformation(SMR) reaction in a continuously stirred tank reactor(CSTR) was used as an example case for exploration of the model reduction technique. Initially, the reaction network of the process, from the work of $\mathrm{Xu}$ and Froment [15], was examined. After consideration of the internal processes involved with the catalyst states, a reduced reaction network was constructed. This excluded the in-between catalyst states of the more elaborate model. Obviously, due to this reduction, details of interactions between the various catalyst states were lost. To account for this, discrepancy terms are introduced to the model.

The intrusive form of model discrepancy correction was used for this work. This ensured that no physical constraints were broken and all possible QoIs were corrected with discrepancy. The model discrepancy terms were incorporated to the physical parameter values. This updated the parameters at each step as the model progressed through the reactions, which is consistent with the physical model of the process. The discrepancy terms themselves were constructed considering the varying order of interactions that the reduced model did not consider. These interaction terms were added incrementally till further additions failed to produce feasible improvements in the model prediction.

\subsection{Steam Methane Reformation Reaction}

The purpose of choosing SMR as example case is due to its importance in the context of today's energy demands. Meeting the ever increasing demands for energy is one of the most important scientific challenges of our time. So far, petroleum and other fossil fuels have been used extensively to meet this demand. However, the increased use of these fuels since the Industrial Revolution has lead to a drastic rise in the overall presence of $\mathrm{CO}_{2}$ in the atmosphere[16]. The negative impact of this and other greenhouse gas byproducts of fossil fuel burning on the environment, along with the fact that we only have a finite source of fossil fuels, have created the need to develop new energy technologies. Ideally, these technologies should use fuel sources other than the remaining deposits of conventional fuels while limiting the repercussions of fuel usage on the environment. One of the more promising of these new sources of energy is the utilization of hydrogen gas as an energy source due to its higher heating values and nearly emission free combustion[17].

Hydrogen gas already has applications across a variety of industries including chemicals, refining industries food processing and pharmaceuticals, but employing hydrogen as a fuel source is still a developing technology[18]. It is also being used increasingly in the energy sector in fuel cells. These fuel cells produce a flow of free electrons using inlet streams of hydrogen and oxygen in the presence of an anode and cathode to produce a flow of free electrons during the formation of water. Fuel cells 
are increasing in popularity and also have practical applications in a wide array of devices. Low temperature fuel cells have been proven effective in the development of hybrid electric vehicles. Higher temperature fuel cells, such as solid oxide fuel cells (SOFCs), are used generally for stationary power generation. Creating electric power and heat from chemical energy, SOFCs have been shown to operate more efficiently than current engines and turbines with a theoretical overall system efficiency of $70 \%$. SOFCs are also favored for producing less pollutants and noise than their counterparts[19]. The vast amounts of hydrogen that exists in nature gives the SOFC great potential as a power source.

However, pure hydrogen, or even easily extract-able hydrogen, is rare in nature. A well known method of hydrogen extraction is electrolysis. In electrolysis, water is split into hydrogen and oxygen using electricity. However, due to the high cost of electricity associated with electrolysis[20,21], this process is not extensively used. Financially, it is more profitable to mass produce hydrogen from methane and other hydrocarbons. Steam methane reformation (SMR) is widely used to produce hydrogen this way due to its cost effectiveness $[17,20]$. SMR is also preferred over other refining processes such as partial oxidation and auto reforming because of its high thermal efficiency and higher ratio of $\mathrm{H}_{2}$ to $\mathrm{CO}_{2}$ concentrations[17,19,20]. Currently, over $75 \%$ of all industrial steam reforming uses natural gas as feed-stock[19]. This is because of methane's high hydrogen content and low initial cost as compared to other sources such as coal[17]. Use of methane as hydrogen can also have an added benefit when the methane is obtained from landfills and other waste sites. Methane emission from landfills amount to about $16 \%$ of all man-made methane emissions[20]. Various methods already exist to extract the methane produced from these sites in an efficient manner [21]. Utilizing this methane means emissions from these sites that would have otherwise accumulated in the atmosphere and contributed to the greenhouse effect can now be used to produce energy in an environmentally friendly manner.

A significant use of steam methane reformation process is when it is used to carry out methane reforming while configured inline with an SOFC to provide a constant inflow hydrogen stream. Steam reforming, given its high thermal efficiency and high operating temperature requirement, is ideal given that current SOFC technology operation requires a high temperature $\left(700^{\circ} \mathrm{C}-1000^{\circ} \mathrm{C}\right)$ [23]. The high operating temperature of the SOFC removes the need for additional heat input to the reformer.[24] Sangtongkitcharoean compared the performance of SOFCs operating with both external and internal reforming showing that direct internal reforming was preferred for its ability to operate at lower values of $F_{\mathrm{H}_{2} \mathrm{O}}$ and $\mathrm{F}_{\mathrm{CH}_{4}}$ [25]. Wang, et. al. examined the impact the operating parameters have on the local temperature and methane conversion for internal reforming, and showed how the heat input from the SOFC boosts performance of the reformer along the length of the reactor[23]. The potential benefit of this inline configuration makes it an option worth exploring further. However, implementing this configuration also necessitates the use of process optimization to develop a more pure hydrogen stream from the reformer while minimizing the additional thermal costs necessary to avoid carbon buildup within the SOFC. Since any process optimization will require the examination of large number of scenarios 
with varying conditions in a short amount of time, this makes creation of an fast computational model for the SMR process critical.

In order to produce a hydrogen stream with the desired attributes for fuel cells, modifications can be made either to the reactor system to improve conversion at given operating conditions or optimizing the operating conditions impacting the kinetics of the reaction to achieve higher $\mathrm{H}_{2}$ production. Most of the time, focus is made on modifying the reactor, primarily due to the ease of implementation since there is still much to learn about kinetics. One promising method for achieving this is inclusion of a palladium based or other hydrogen permeable membranes to separate hydrogen from the resultant gas mixture[1, 26, 6, 7]. Investigations have shown that the use of the membrane creates a greater conversion of methane and hence faster production of hydrogen $[1,26]$. But the extra cost of preparation of the membrane makes this method's implementation on an industrial scale unfeasible. Other similar methods to increase hydrogen production often include the addition of either an oxygen secondary reformer or a pre-reformer into the overall reactor design. A secondary oxygen reformer combusts unused methane allowing for more feed gas to be processed without a loss to product purity. However, this configuration does require additional downstream treatments to handle the greater influx of $\mathrm{CO} 2$ produced. A pre-reformer allows for a portion of the hydrocarbon to be reformed before entering the main reactor, and is the product of the development of high activity catalysts[27]. These are able to achieve reforming at lower temperatures and have been shown to be effective at increasing overall hydrogen production by Bavarsad[27, 19]. To be effective though, this high activity catalyst requires a much higher nickel content and special treatment to have higher surface area[27].

A key area of interest that is often ignored in the optimization of SMR is the reaction kinetics. This is primarily due to the lack of knowledge regarding the reaction kinetics. Studies into the kinetics of SMR date as far back as the 18th century. However, the first thorough investigations were published as late as the 1950's[28, 29]. Van Hook provides a comprehensive summary of the early investigations of SMR (1933-1952) including some introductory research into the governing kinetics[30]. Akers and Camp provide one of the earliest comprehensive investigations into SMR[31]. Their work investigated the kinetics over a nickel catalyst for a range of temperatures and pressures. Experimental testing along with a thermodynamic analysis of a proposed network of gas phase reactions revealed that the reaction is first order with respect to methane, and that carbon monoxide and carbon dioxide are primary products with carbon dioxide being produced at a much faster pace. Additionally, it was shown that the overall process is equilibrium limited so additional modifications are required for increasing the yield of $\mathrm{H}_{2}$. These information were extensively used in the creation of the reduced model for this work.

Ross and Steel explored the variability of the catalyst and its impact on the reaction kinetics. Through systematic variations of the catalyst composition, data was collected on the impact on the kinetics and stoichiometry under these slightly altered conditions[32]. Munster and Grabke built up on this work, examining SMR on various iron, nickel and iron-nickel alloy catalysts. A flow reactor operating under standard conditions was used to develop a functional relation between the reaction 
rates and catalyst activity. Deactivation of the catalyst due to carbon deposits and its impact on kinetics were also examined.[33].

However, to date, the work of $\mathrm{Xu}$ and Froment has proven to be the most thorough study on steam reforming, and has become the basis for much of the modern research on the subject[34]. The attention paid to the kinetics and their importance in the reforming process is the main distinction separating this work from previous work. Until this point, $\mathrm{Xu}$ and Froment believed that the role of kinetics in proposed simulations is either neglected or very over simplified[28]. Starting with a set of eleven possible reactions involving the main gas phase constituents, a thermodynamic analysis was conducted to eliminate all those incapable of propagating the reactions leaving the following three main gas phase reactions (GPR):

I) $\mathrm{CH}_{4}+\mathrm{H}_{2} \mathrm{O} \rightleftharpoons \mathrm{CO}+3 \mathrm{H}_{2}$

II) $\mathrm{H}_{2} \mathrm{O}+\mathrm{CO} \rightleftharpoons \mathrm{CO}_{2}+\mathrm{H}_{2}$

III) $\mathrm{CH}_{4}+2 \mathrm{H}_{2} \mathrm{O} \rightleftharpoons \mathrm{CO}_{2}+4 \mathrm{H}_{2}$
$\Delta H=206.1 \mathrm{~kJ} / \mathrm{mol}$

$\Delta H=41.15 k J / m o l$

$\Delta H=165.0 \mathrm{~kJ} / \mathrm{mol}$

Reaction schemes were then developed based on these GPR to more accurately detail how the process advances from reactants to products in the presence of a catalyst. Several guidelines based on literature findings were adhered to for the creation of the differing schemes. These step by step guidelines govern the interaction of steam and methane with the catalyst and the behavior of the interaction between the radicals:[15]

1. $\mathrm{H}_{2} \mathrm{O}$ reacts with surface atoms of the catalyst, producing adsorbed oxygen and gaseous hydrogen.

2. Methane is adsorbed on surface atoms. The adsorbed methane either reacts with the adsorbed oxygen or is dissociated to form chemisorbed radicals, such as $\mathrm{CH}_{3}$, $\mathrm{CH}_{2}^{*}, \mathrm{CH}^{*}$ and $\mathrm{C}^{*}$.

3. The concentrations of the carbon-containing radicals $\mathrm{CH}_{3}^{*}, \mathrm{CH}_{2}^{*}, \mathrm{CH}^{*}$ and $\mathrm{C}^{*}$ are much lower than the total concentration of the active sites.

4. The adsorbed oxygen and the carbon containing radicals react to form $\mathrm{CH}_{2} \mathrm{O}^{*}, \mathrm{CHO}^{*}, \mathrm{CO}^{*}, \mathrm{CO}_{2}^{*}$.

5. The hydrogen formed is directly desorbed into the gas phase and/or the gaseous hydrogen is in equilibrium with $H^{*}$ or $H_{2}^{*}$.

6. Each reaction scheme has one "rate determining" step relating to equations I, II, and III with a rate potentially slower than all others, so that it controls the overall reaction.

Following these guidelines a reaction scheme was proposed allowing for parallel formation of $\mathrm{CO}$ and $\mathrm{CO} 2$ out of the adsorbed radical $\mathrm{CHO}$ as shown in Figure 1. 


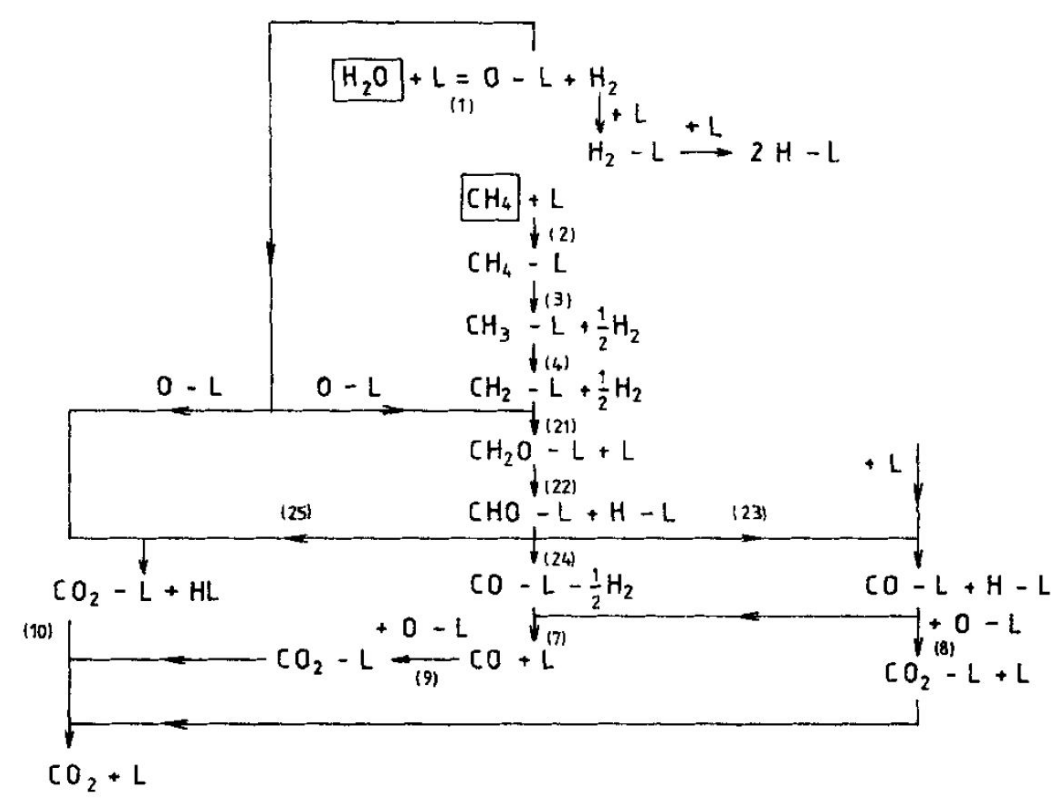

Figure 1: Xu-Froment Proposed Reaction Scheme[15]

This scheme equates to the 13 reaction mechanism of elemental steps listed in Equations A-M.[15]

$$
\begin{aligned}
& \mathrm{H} 2 \mathrm{O}+\mathrm{L}=\mathrm{O}^{*}+H 2 \\
& \mathrm{CH} 4+\mathrm{L}=\mathrm{CH}_{4}^{*} \\
& \mathrm{CH}_{4}^{*}+L=C H_{3}^{*}+H^{*} \\
& \mathrm{CH}_{3}^{*}+L=C H_{2}^{*}+H^{*} \\
& \mathrm{CH}_{2}^{*}+O^{*}=C H 2 O^{*}+L \\
& \mathrm{CH}_{2} \mathrm{O}^{*}+L=C H O^{*}+H^{*} \\
& \mathrm{CHO}^{*}+L=C O^{*}+H^{*} \\
& \mathrm{CO}^{*}+O^{*}=C O_{2}^{*}+L \\
& \mathrm{CHO}^{*}+O^{*}=C O_{2}^{*}+H^{*} \\
& \mathrm{CO}^{*}=C O+L \\
& \mathrm{CO}_{2}^{*}=C O 2+L \\
& 2 \mathrm{H}^{*}=H_{2}^{*}+L \\
& \mathrm{H}_{2}^{*}=H 2+L
\end{aligned}
$$

This kinetic network has been one of the most extensively applied to simulate industrial reactors operating under standard conditions. This is due to the generality of the framework which leads to greater reliability in results under varying conditions. Also, it helped to resolve contradictions surrounding the reaction order with respect 
to steam[35]. This proposed kinetic network has also been validated with previous works. The work of Ding and Alpay showed that this network is suitable for predicting transient response making it one of the few models capable for use in process design[28]. This limit in the number of transient capable networks has slowed the development of optimization algorithms, but advances in high performance computing has opened the door for the use of more powerful solving methods to address the problem. Achieving process design and optimization through simulation would allow for the production of predictions without the complications associated with physical testing. Rajesh, et. al. demonstrated this with the usage of a genetic algorithm for the multi-objective purposes of minimizing the methane in-flow for a given hydrogen output and maximizing the amount of carbon monoxide present in the synthetic gas.[36] The creation of more computationally tractable SMR model will facilitate faster and better optimizations. However simulating more intricate systems including inline configurations or separation membranes would require taking into account other physical processes such as fluid flow and heat transfer. This would make the process computationally unfeasible. A reduced cheaper model of SMR kinetics that also uses discrepancy terms to incorporate the effects of these physical process into the model may provide a solution to this problem.

There are a number of problems with computationally simulating the SMR process. Simulation grants the flexibility of application to a large number of reactor designs operating at an infinite set of conditions, but variability of the kinetic mechanism and intense computational needs has hindered its use for SMR[17, 29]. Both the value of associated kinetic parameters as well as the overall structure of the kinetic model are highly sensitive to the composition of the catalyst[32]. This makes it very difficult to develop a generalized set of equations applicable to all catalyst types and compositions through a simple change in variables[29]. Because of this, a variety of mechanisms have been created to simulate SMR across a wide range of temperatures $(260-1000)^{\circ} \mathrm{C}$ and pressures $(100-5000) \mathrm{KPa}[30,35]$. Simplifying this kinetics issue would greatly simplify the problem. Using statistical model building to capture the effect of the catalyst on the structure of the kinetics model, using experimental data as indicators, as demonstrated in this work, may be an option.

The other main drawback with the simulation of SMR is the high computational demand required to produce an accurate, realistic simulation. Methane reformation is the combination of a catalytic reactor, fluid dynamics, and heat transfer problem. Incorporating all this into a single model creates a simulation requiring an execution time that is unfeasible. Using improved analytic methods, existing simulation methods can be advanced to improve overall quality of results while maintaining or improving the computational efficiency. Traditional modeling methods for catalytic reactions are usually built using the Langmuir Hinshelwood kinetics. In this method, the kinetics are based on establishing a rate determining step which dictates the progression of the overall reaction; however, application of this to SMR is not simple. The rate determining step tends to change depending on the percentage of nickel in the catalyst[33], as demonstrated by Munster's work . Xu and Froment addressed this 
issue by selecting a rate determining step for each of the gas phase reactions and then building the reaction rates. The accuracy of their model was displayed in recreating the results of De Deken's study examining the behavior of a industrial sized reformer while taking into account diffusion limitations[37].

The proposed reduced order modeling methodology attempts to explore a new method of model reduction while addressing the current issues associated with computational simulation of SMR. The preliminary inaccurate reduced model is first obtained by minimizing the reaction network in Equations I-XIII using Taken's theorem. This minimizes the total number of necessary ODEs required to create a valid simulation. These simplifications induce additional uncertainty driving the model further from the reality. Stochastic discrepancy functions are then embedded in model parameter expressions to correct for this bias. A joint normal prior distribution is assumed for both physical parameters and discrepancy parameters, named $\beta$ and $\theta$ parameters respectively. Then, Bayesian calibration is used to calibrate the model to data obtained from the work of $\mathrm{Xu} \&$ Froment. The Bayesian calibration routine evaluates the likelihood of a given set of parameters given how closely the model matches with the data. This likelihood is utilized by a Markov Chain Monte Carlo(MCMC) routine to search the parameter space and eventually create a posterior distribution of parameters. Using the posterior distribution, a number of model predictions are made and the model is tested for interpolation. After validating the interpolation capabilities of the final model, it is extended to industrial scale. 


\section{Model Development}

\subsection{Model Reduction}

Reducing the number of equations from the $\mathrm{Xu}$ and Froment reaction network requires a systematic approach to determine which chemical species are essential. After this is determined, the reactions involving the non-essential chemical species can be omitted. This omission is carried using the principles described by Takens's theorem. The dynamic system reduction technique suggested by Takens's theorem proposes that a dynamic system can be reconstructed from the time series of a variable that is coupled to all the states in the system. To illustrate an example, take the very simple reaction network detailing the creation of species $\mathrm{H}$ from system reactants $\mathrm{A}$ and $\mathrm{D}$ :

$$
\begin{gathered}
\mathrm{A}=\mathrm{B}+\mathrm{C} \\
\mathrm{C}+\mathrm{D}=\mathrm{E}+\mathrm{F} \\
\mathrm{B}+\mathrm{F}=\mathrm{G} \\
\mathrm{E}+\mathrm{G}=\mathrm{H}
\end{gathered}
$$

The above reaction network can be reduced to a simpler form now using Taken's theorem. The principle of Taken's theorem allows for the reduction of dynamic networks based on causal relationships without the loss of vital information. Creating a reaction graph based on the network mechanism illustrates the dependencies of the different species and allows for quicker identification of the desired causal relationships. Connections between the different species are made with lines between nodes representing the listed chemical reactions. This provides a more clear way of visualizing how the system progresses from start to finish, and is necessary when determining the species required by the reduced model. The next figure, from the thesis of Ford[38] shows this concept applied to the idealized system above. 


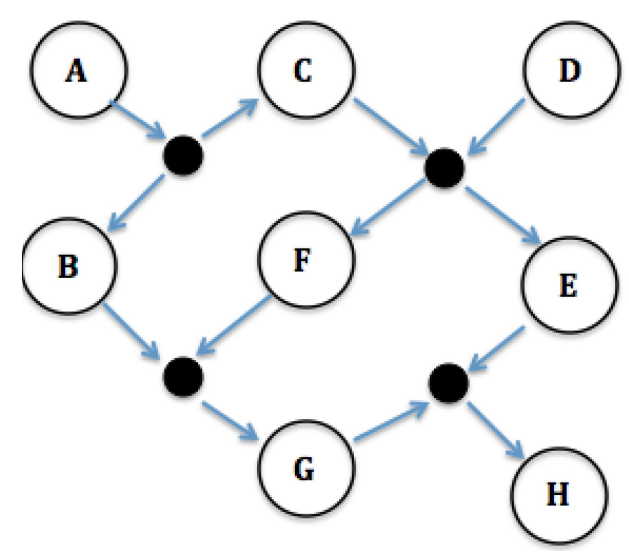

Figure 2: Idealized Full Reaction Network[38]

With the network graph in place, intermediate species can be quickly identified. In this case, species B, C, E, F, and G are all intermediate states since they originated from species introduced before them. Therefore, they can be removed without significant change to the core structure of the system, since information about their impact is contained in the time series of $\mathrm{A}, \mathrm{D}$ and $\mathrm{H}$. A minimal set of components containing the variability of the full network can now be determined through exploiting the various causal relationships. For example, species A, B, and $\mathrm{G}$ are related given that A reacts to create $B$ which reacts a second time to create $G$. A is the only reactant to the reaction producing $B$. This indicates that all the information regarding species $B$ is contained within the time series of species A, so a relation directly linking A to $G$ can be created combining the kinetics of two original reactions. This simplification makes species B unnecessary to describe evolution of $G$ in the system.

Reductions such as this are done systematically until all intermediate states are removed leaving a streamlined network graph shown in figure below.

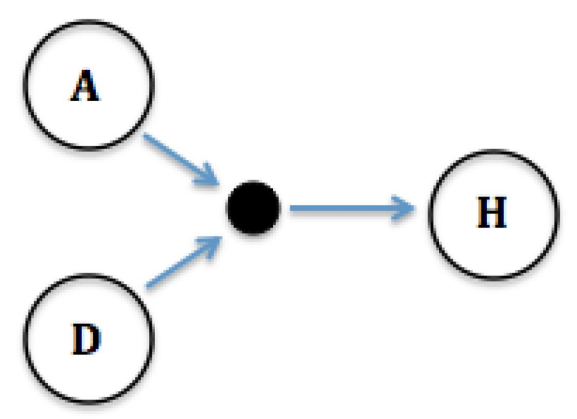

Figure 3: Reduced Reaction Network[38] 
Making network reductions in this manner should ideally maintain the full variability associated with the original expanded model. In the simplified example illustrated above, the information about the intermediate states is contained with in the time series for species A, D, and H. This enables the dynamic system to be simplified in the following manner.

Making simplifications in this manner leads to more information incorporated into fewer variables. The addition of the discrepancy functions works to extract this condensed information by introducing additional variability to the reaction parameters. This allows the reduced configuration to match the behavior of the higher fidelity network despite the limited number of total variables.

This model reduction methodology was applied to the $\mathrm{Xu}$ and Froment network to determine the minimum number of essential states to describe the Steam Methane Reformation process and to produce the proposed reduced order model. A reaction graph of the entire process including the catalyst states was created in a manner similar to that of the idealized example. The 16 gas phase and adsorbed species are all related into the mapping of the "flow" of the reaction from reactants to products. The figure below, from the thesis of Ford[38], depicts this full network graph. Nitrogen is not included despite being in the tank because it is inert in the working conditions of the reactor and hence do not take part in any of the chemical reactions.

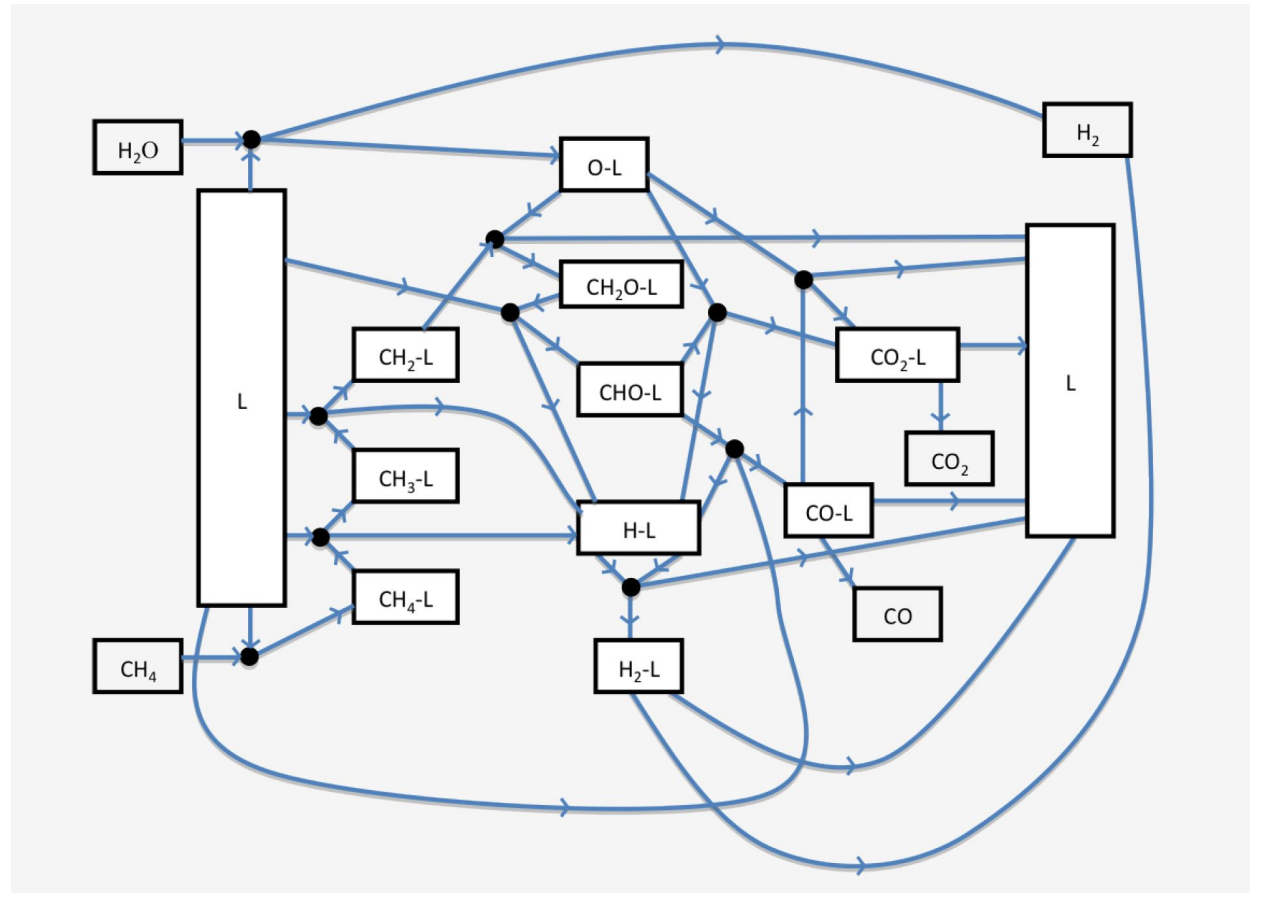

Figure 4: $\mathrm{Xu}$ and Froment Full Reaction Network[38]

Inspection of the reaction graph reveals several simplifications that can be clearly made. For example, $\mathrm{CH}_{4}$ can be related to its adsorbed radicals $\mathrm{CH}_{4}-\mathrm{L}_{2} \mathrm{CH}_{3}-\mathrm{L}$, and $\mathrm{CH}_{2}-\mathrm{L}$; gas phase products can be linked to their adsorbed counter- parts and $\mathrm{CH}_{2} \mathrm{O}-\mathrm{L}$ can be removed from the dynamic system by collapsing the in between node. However, not all simplifications will be so apparent and may require careful investi- 
gation of the network's structure. Fully simplifying the system leaves a streamlined network dependent on only the gas phase species present in steam methane reforming reaction network as shown in the next Figure. Hence, the presence of the catalyst and its absorbed states are removed from the model of the dynamic system. But their information is still contained within the time series of the gas species. This reduces the number of ODEs representing the systems, making the model much more inexpensive computationally.

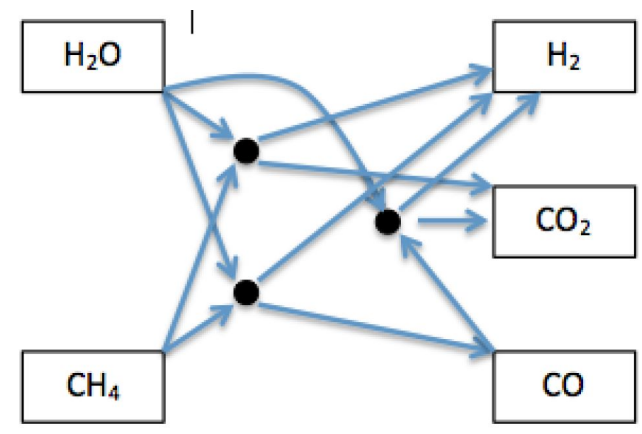

Figure 5: Xu and Froment Reduced Reaction Network[38]

The remaining species form the basis around which the kinetics of the ROM are formed. This new kinetic model will have to account for all the information lost due to the removal of species as a result of the simplifications. This will be done by incorporating dynamic discrepancy into the model. The discrepancy functions will take as inputs the gas phase species. The time series of these gas phase species contains the information about omitted states. This will enable discrepancy functions to recreate the variability of the full model.

This particular method of model reduction can be applied to numerous dynamic systems with intermediate states that are not the quantity of interest. This makes the choice of this approach as a path towards making the reduced order model generic. However, depending on the specific process and its dynamic model, alternative methods of reduction can also be applied. As Petzold and Zhu discussed in their work, various techniques of reduction has been used over the years on chemical reaction networks[26]. These involved applying steady-state approximation to the appropriate radicals, the partial-equilibrium approximation to the fast reversible reactions and ignoring the very slow and therefore unimportant reactions. Another approach involved carrying out a sensitivity analysis. Defining as parameters the rate constants of the chemical reactions, sensitivity analysis determines the change in the species concentration for small perturbations of the rate constants. If a reaction is slow and has minimal effect on the QoI(Quantity of Interest), it can be identified in this way.

Likewise, similar field-specific reduction techniques will exist for dynamic systems in different fields. The method described by subsequent sections to model the discrepancy and uncertainty arising from reduction of the model will apply to all these different scenarios since the model discrepancy terms will be generic and solely based on the model inadequacy introduced by model reduction. This will make this approach generally applicable. 


\section{$2.2 \quad$ Model Kinetics}

The reduction in state variables from 16 to 6 necessitates the development of a new reaction network and kinetic system to better suit the nature of the minimal set of states in the reduced order model. The lack of adsorbed radicals allows for the use of the three gas-phase reactions (Equations I, II, and III) for this purpose, but additional measures are required to account for the induced error associated with the dynamic model reduction. In many ways, the structure of the reduced model mimics that of the high fidelity model, especially in the general form of the mass balance ODEs and the applied rate expressions. The model is described using a set of ODEs describing the partial pressure of each constituent species. The total pressure of all species inside the tank is constant. Hence, using the partial pressure in the ODEs gives the option to validate the code as well as the mathematical model by checking the sum of pressures of all the species inside the reactor. If the total pressure is above or below the initial pressure, there is an error with the computation or the mathematical model.The general form of the pressure balance equations after stoichiometry was taken into account is as follows:

$$
\begin{gathered}
\frac{d P_{C H 4}}{d t}=\left(P_{a t m}\right)^{-1}(R T / V)\left(N_{C H 4}-V r_{1}-V r_{3}-X_{C H 4}\left(N_{O U T}\right)\right) \\
\frac{d P_{H_{2} O}}{d t}=\left(P_{a t m}\right)^{-1}(R T / V)\left(N_{H 2 O}-V r_{1}-V r_{2}-2 V r_{3}-X_{H 2 O}\left(N_{O U T}\right)\right) \\
\frac{d P_{C O_{2}}}{d t}=\left(P_{a t m}\right)^{-1}(R T / V)\left(V r_{2}+V r_{3}-X_{C O 2}\left(N_{O U T}\right)\right) \\
\frac{d P_{H_{2}}}{d t}=\left(P_{a t m}\right)^{-1}(R T / V)\left(3 V r_{1}+V r_{2}+4 V r_{3}-X_{H 2}\left(N_{O U T}\right)\right) \\
\frac{d P_{C O}}{d t}=\left(P_{a t m}\right)^{-1}(R T / V)\left(V r_{1}-V r_{2}-X_{C O}\left(N_{O U T}\right)\right)
\end{gathered}
$$

where

$P_{a t m}=$ atmospheric pressure in SI Units $(\mathrm{Pa})$

$r_{1}, r_{2}, r_{3}=$ rates of gas phase reactions I,II and III respectively

$X_{\mathrm{CH}_{4}}=$ partial pressure fraction of methane

$V=$ volume of reactor $\left(m^{3}\right)$

$T=$ temperature $(\mathrm{K})$

$R=$ Universal Molar Gas Constant $\left(\mathrm{J} \cdot \mathrm{mol}^{-1} K^{-1}\right)$

$N_{\text {OUT }}=$ Total molar outflow in $\mathrm{mol} / \mathrm{s}$.

The above equations can be derived using some assumptions about the process, as will be demonstrated now. The SMR reactions take place in a continuously stirred tank reactor(CSTR). A typical CSTR is illustrated in the next figure[39]. 


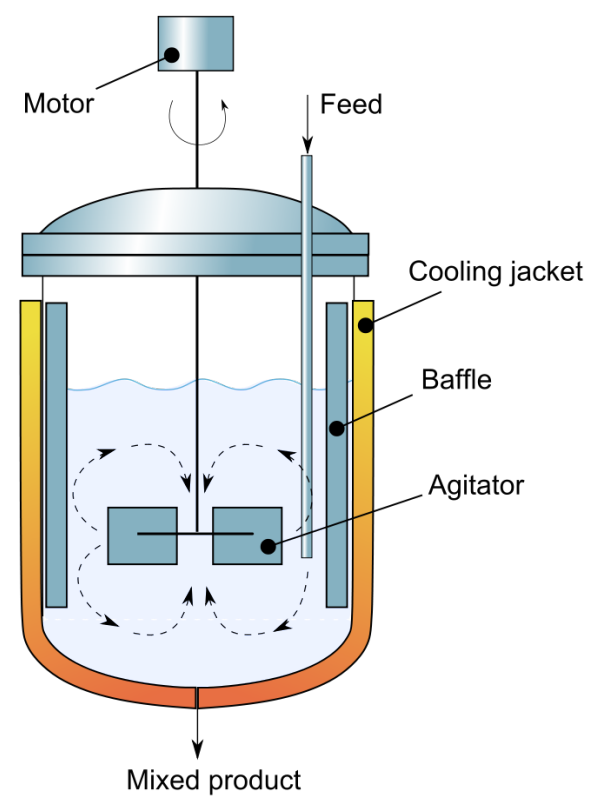

Figure 6: CSTR[39]

As can be seen in the figure, feed continuously enters the tank while the mixed product continuously exits it. An agitator ceaselessly stirs the contents of the tank during its operation. When modeling a process inside a CSTR, the following simplifying assumptions can be made:

I ) Perfect or ideal mixing

II) Constant Homogeneous Density

III) Isothermal Conditions

IV) Steady-state Conditions After a Certain Amount of Time

V) Total Inflow Equals Total Outflow in $\mathrm{mol} / \mathrm{s}$

The last assumption equating total molar inflow to total molar outflow enables the development of an overall mass balance equation in the tank. In its most generic form, this assumption translates to the following equation.

$$
[\text { accumulation }]=[\text { in }]-[\text { out }]+[\text { generation }]=0
$$

[generation] or the increase in number of moles inside the tank due to reactions can be defined in terms of the rates of the gas phase reactions.

$r_{1}, r_{2}, r_{3}=$ moles of $\mathrm{CH}_{4}$ consumed by GPRs I,II and III for unit volume and time [generation] $=\left(2 V r_{1}+2 V r_{3}\right)$

where the coefficient " 2 " is the net difference in moles between reactants and products for gas phase reactions I and III. 
The expression for accumulation inside the tank becomes

$$
[\text { accumulation }]=\text { inflow }- \text { outflow }+\left(2 V r_{1}+2 V r_{3}\right)=0
$$

where $2 V r_{1}$ and $2 V r_{3}$ are net generation of moles for each mole of reactants used up in the reactions I and III respectively. Since accumulation in the tank is zero, the above relation produces the following expression for total outflow from tank.

$$
\begin{gathered}
\text { outflow }=\text { inflow }+\left(2 V r_{1}+2 V r_{3}\right) \\
\text { outflow }=N_{C H 4}+N_{H 2 O}+\left(2 V r_{1}+2 V r_{3}\right) \\
\text { outflow }=N_{O U T}=N_{\text {TOTAL }}+\left(2 V r_{1}+2 V r_{3}\right)
\end{gathered}
$$

The feed to the reactor consists of methane and steam inflows only and can be summed $N_{\text {TOTAL }}$. So, using the total molar outflow, the outflow of each species can be found by simply multiplying it with the value of the partial pressure fraction. For instance for methane

$$
\frac{d N_{C H 4}}{d t}=[\text { inflow }+ \text { generation }]-\frac{P_{C H 4}}{P_{\text {Total }}}\left(N_{\text {TOTAL }}+\left(2 V r_{1}+2 V r_{3}\right)\right)
$$

where

$$
\begin{gathered}
P_{C H_{4}}=\text { Methane pressure inside tank } \\
P_{\text {Total }}=\text { Total pressure inside tank }
\end{gathered}
$$

Modifying the above equation for rate of change of pressure gives

$$
\frac{d P_{C H 4}}{d t}=\left(P_{a t m}\right)^{-1}(R T / V)\left(N_{C H 4}-V r_{1}-V r_{3}-X_{C H 4}\left(N_{T O T A L}+\left(2 V r_{1}+2 V r_{3}\right)\right)\right)
$$

In a similar way, the expressions for other species are derived. Eventually, we obtain the set of equations in equations 5,6,7,8 and 9. Substituting the $N_{\text {OUT }}$ variable with the relevant expression above, the model equations are established.

The next step would be to determine expressions for the rates of gas-phase reactions(GPR). They are as follows

$$
\begin{aligned}
& r_{1}=k_{1}\left\{P_{C H 4} \cdot P_{H 2 O}-\frac{P_{C O} \cdot P_{H 2}^{3}}{\kappa_{1}}\right\} \\
& r_{2}=k_{2}\left\{P_{C O} \cdot P_{H 2 O}-\frac{P_{C O 2} \cdot P_{H 2}}{\kappa_{2}}\right\} \\
& r_{3}=k_{3}\left\{P_{C H 4} \cdot P_{H 2 O}^{2}-\frac{P_{C O 2} \cdot P_{H 2}^{4}}{\kappa_{3}}\right\}
\end{aligned}
$$


where

$$
\begin{gathered}
k_{1}, k_{2}, k_{3}=\text { rate constants of reactions I,II and III } \\
\kappa_{1}, \kappa_{2}, \kappa_{3}=\text { equilibrium constants of reactions I,II and III }
\end{gathered}
$$

From the examination of past literature on the kinetics of SMR, GPR II can be theoretically assumed to be infinitely fast compared to the other two gas-phase reactions. It can be assumed to be always in equilibrium. Hence, ideally, we would not have to solve the system for $P_{C O}$ since the species involved in GPR II would be related by the following relation at all times.

$$
\begin{gathered}
\kappa_{2}=\frac{\left(P_{\mathrm{CO} 2}\right)\left(P_{\mathrm{H} 2}\right)}{\left(P_{\mathrm{CO}}\right)\left(P_{\mathrm{H} 2 \mathrm{O}}\right)} \\
P_{\mathrm{CO}}=\frac{P_{\mathrm{CO} 2} \cdot P_{H 2}}{\kappa_{2} \cdot P_{\mathrm{H} 2 \mathrm{O}}}
\end{gathered}
$$

The value of $P_{C O}$ can be obtained by simply putting in the values of all the other variables in this equation. However, solving the system in this manner proved to have a disadvantage. Since a parametric calibration requires the initial assumption about the parameters to have wide range, $\kappa_{2}$ will often have values much smaller than one. Since $\kappa_{2}$ appears in the denominator of the expression for $P_{C O}$, this will often lead to values of $P_{C O}$ much larger than the total pressure inside the tank, resulting in solver failure.

To counter this problem, instead of assuming GPR 2 to be infinitely fast and always at equilibrium, a very large value of $k_{2}$ is assumed instead. This leads to the addition of a state to the model but solves this particular problem involving calibration and solver failure. If a reasonable bound can be known for $\kappa_{2}, P_{C O}$ can be removed from the system, reducing the number of states to 4 from 16 .

Since the data used for calibration is for isothermal SMR occurring at different constant temperatures, temperature dependent expressions were used for the physical parameters $k_{1}, k_{2}, k_{3}, \kappa_{1}, \kappa_{2}$ and $\kappa_{3}$.

For rate constant, Arrhenius's equation was used.

$$
k=A e^{-E_{a} / R T}
$$

where 


$$
\begin{gathered}
A=\text { Pre-exponential Factor } \\
E_{a}=\text { Activation Energy } \\
T=\text { Absolute Temperature } \\
R=\text { Universal Molar Gas Constant }
\end{gathered}
$$

The equilibrium constant $\kappa$, is defined by the following expression:

$$
\kappa=\left(e^{\Delta S / R}\right)\left(e^{-\Delta H / R T}\right)
$$

where

$$
\Delta S=\text { Change in Entropy }
$$

Essentially, the Reduced Order Model(ROM) is constructed from applying the basic knowledge of chemical kinetics to the three gas phase reactions obtained after reducing the reacting network. After its basic construction though, it is augmented with the embedding of the discrepancy terms in the expressions for the physical parameters, i.e, the rate constants and the equilibrium constants. The incorporation of the discrepancy terms take the following generic form.

$$
\begin{aligned}
k & =k e^{\left(\delta_{k}\right)} \\
\kappa & =\kappa e^{\left(\delta_{\kappa}\right)}
\end{aligned}
$$

In the above expressions, $\delta$ is the value of the discrepancy function corresponding to that particular physical parameter. The two equations above update the parameter values at each iteration of the solver. Hence, the values of the physical parameters are updated with time as the system changes, introducing the lost variability of the actual reaction mechanism. How the discrepancy terms are obtained and calculated is described in the subsequent sections.

\subsection{Model Solver}

As discussed before, the mathematical model for the SMR process will consist of a collection of Ordinary Differential Equations(ODEs). This is will be the case for both the skeletal reduced model and the reduced model with discrepancy. In the case of the model augmented with discrepancy, additional terms will be attached to the expressions for the model parameters, which will update the parameters as the system moves from one point to another.

A preliminary attempt to solve the ODE system for the particular configuration at hand showed that the ODE system was stiff in nature. This was demonstrated when the "ode45" function in MATLAB took excessively large amounts of time to solve the system. Stiff ODE systems undergo very rapid changes in certain time regions but behave quite normally in other regions. Hence, solving stiff ODEs efficiently requires 
a solver with adaptive step size. The MATLAB function "ode15s" proved to be very efficient for this purpose. However, since each Bayesian Calibration of the model will require a minimum of 50000 model runs and arriving at an optimum model may require tens or even hundreds of calibrations, MATLAB, being a compiled language and hence considerably slower, was not suitable for this purpose. Also, because MATLAB was proprietary, recreating or building on this work in MATLAB will be problematic without the appropriate license. Hence, $\mathrm{C}++$ was used for running the models for this work. The ODE systems of the model were solved using the $\mathrm{C}++$ ODE solver ODEINT. A variety of solution methods were available from the ODEINT library. For the stiff system describing the reduced model, the Rosenbrock method was used for solution. The multi-step Rosenbrock method proved to be an efficient solver for this work, solving each model run with a minimum relative accuracy of $10^{-3}$ in a fraction of a second.

\subsection{Discrepancy Function}

The dynamic discrepancy terms consist of two parts: a set of deterministic basis functions and a vector of stochastic coefficients. The state variables of the model, which, for the SMR model, are the the partial pressures, act as the inputs to the basis functions. This is consistent with Takens's Theorem, which states that the time series of the coupled states contain information about the evolution of the state space variables[40][41]. The state variables of the model, used as inputs to the basis functions at different times, are essentially the time series of the coupled states. Encapsulated in the variation of these state variables is the information regarding the omitted species. Combined, these terms together quantify the uncertainty resulting from the reduction of the model. This enables the reduced order model to match the behavior of the high fidelity model. The generic form of the discrepancy term is shown in the equation below.

$$
\delta=\sum \beta_{a, i} \phi_{i}\left(x_{a}\right)+\sum \beta_{a, i, b, j} \phi_{i}\left(x_{a}\right) \phi_{j}\left(x_{b}\right)+\ldots
$$

Here

$\delta=$ value of the discrepancy term

$\phi_{i}=$ basis function of order ' $\mathrm{i}$ '

$x_{i}=$ the $i^{\text {th }}$ component of input vector $\mathrm{X}$

$\beta_{a, i}=$ the stochastic coefficient $\beta$ corresponding to basis function $\phi_{i}$ with $\mathrm{a}^{\text {th }}$ input $\beta_{a, i, b, j}=\beta$ for basis functions $\phi_{i}$ and $\phi_{j}$ with $\mathrm{a}^{t h}$ and $\mathrm{b}^{\text {th }}$ input

The discrepancy terms are built piece-wise for each physical parameter, examining which state variables influence a specific parameter. These state variables will 
act inputs to the discrepancy function corresponding to that particular physical parameter.Once these inputs are determined, the interactions of the state variables are taken into account to create the discrepancy functions. Interactions can be of varying orders. First order interactions, generally called 'main effects', simply involve a single state variable. Second order interactions or two-way interactions, involve two state variables. This pattern is extended for interactions for higher order. This is demonstrated now with the physical parameter $\kappa_{1}$, the equilibrium constant of reaction I, as example.

$$
\text { I) } \mathrm{CH} 4+\mathrm{H} 2 \mathrm{O} \rightleftharpoons \mathrm{CO}+3 \mathrm{H} 2 \quad \Delta H=206.1 \mathrm{~kJ} / \mathrm{mol}
$$

Since $\mathrm{CH}_{4}, \mathrm{H}_{2} \mathrm{O}, \mathrm{CO}$ and $\mathrm{H}_{2}$ are the participants of reaction I, the partial pressures of these species, which are state variables of the model, will act as inputs to the discrepancy function for $\kappa_{1}$. Since $\kappa_{1}$ is also temperature dependent, $\frac{1}{T}$ will be fifth input. If all main effects are taken into account, the discrepancy function will have the following form:

$$
\delta=\sum_{a, i}\left(\beta_{a, i}\right) \phi_{i}\left(x_{a}\right)=\sum_{i}\left(\beta_{1, i}\right) \phi_{i}\left(x_{1}\right)+\sum_{i}\left(\beta_{2, i}\right) \phi_{i}\left(x_{2}\right)+\ldots+\sum_{i}\left(\beta_{5, i}\right) \phi_{i}\left(x_{5}\right)
$$

where

\begin{tabular}{||cc||}
\hline Symbol & Variable \\
\hline \hline$x_{1}$ & $P_{C H_{4}}$ \\
\hline$x_{2}$ & $P_{H_{2} O}$ \\
\hline$x_{3}$ & $P_{C O}$ \\
\hline$x_{4}$ & $P_{H_{2}}$ \\
\hline$x_{5}$ & $(1 / T)$ \\
\hline
\end{tabular}

Table 1: Discrepancy Function Inputs for $\kappa_{1}$

Hence, equation 17 above translates to

$\delta=\sum_{a, i}\left(\beta_{a, i}\right) \phi_{i}\left(x_{a}\right)=\sum_{i}\left(\beta_{C H_{4}, i}\right) \phi_{i}\left(P_{C H_{4}}\right)+\sum_{i}\left(\beta_{H_{2} O, i}\right) \phi_{i}\left(P_{H_{2} O}\right)+\ldots+\sum_{i}\left(\beta_{1 / T, i}\right) \phi_{i}(1 / T)$

where $x_{a}$ with a $=1,2,3,4$ and 5 will correspond to $P_{C H_{4}}, P_{H_{2} O}, P_{C O}, P_{H_{2}}$ and $\frac{1}{T}$. The subscripts used with $\beta$ indicate which term they correspond to in the discrepancy function. For instance $\left(\beta_{C H_{4}, 2}\right)$ will correspond to a main effect with basis function of order 1 and $P_{C H 4}$ as input. Hence, its term will be $\left(\beta_{C H_{4}, 2}\right) \phi_{2}\left(P_{C H 4}\right)$. In equation 
18 , the terms $\sum_{i}\left(\beta_{C H_{4}, i}\right) \phi_{i}\left(P_{C H_{4}}\right), \ldots \sum_{i}\left(\beta_{1 / T, i}\right) \phi_{i}(1 / T)$ correspond to all the maineffects terms for $\mathrm{CH}_{4}, . .1 / \mathrm{T}$ respectively. These terms can be further expanded in terms of the order of basis functions used as shown.

$$
\sum_{i}\left(\beta_{C H_{4}, i}\right) \phi_{i}\left(P_{C H_{4}}\right)=\left(\beta_{C H_{4}, 1}\right) \phi_{1}\left(P_{C H_{4}}\right)+\left(\beta_{C H_{4}, 2}\right) \phi_{2}\left(P_{C H_{4}}\right)+\ldots
$$

where $\phi_{1}$ and $\phi_{2}$ correspond to basis functions of order 1 and 2 respectively. In this manner, basis functions of varying order can be incorporated into the discrepancy function. As the order of the basis functions increases, its impact on the discrepancy function and hence the model output decreases. This is discussed later.

If second order interactions or two-way interactions are considered, the following term is added to the expression for discrepancy.

$$
\sum\left(\beta_{a, i, b, j}\right) \phi_{i}\left(x_{a}\right) \phi_{j}\left(x_{b}\right)
$$

where $\mathrm{a}=1-5, \mathrm{~b}=1-5$ and $\mathrm{a} \neq \mathrm{b}$. In the above expression $\mathrm{i}$ and $\mathrm{j}$ correspond to the order of the basis functions. The subscripts $\mathrm{a}$ and $\mathrm{b}$ for $x_{a}$ and $x_{b}$ refer to the inputs used. As with the main effects, the subscripts used with the $\beta$ parameter value indicate which term it corresponds to by indicting the order of basis functions used and inputs used for the term. There will be 6 two-way interactions for $\kappa_{1}$, for all possible two combinations of the state variables. In a similar say, higher order interactions can also be incorporated into the discrepancy terms. Combining higher order discrepancy terms will augment the accuracy of the final model at the cost of computational complexity. However, after adding a certain number of terms, the gain in accuracy of the model will start to decrease compared to the increase in complexity of the model. That is why discrepancy terms are added incrementally. After each addition, the accuracy of the model is observed and the corresponding gain in computational complexity is examined.

In addition to the order of interactions included, the other factor influencing the shape of the discrepancy functions is the order of basis functions included. The order of a particular basis function is indicated by its $\operatorname{subscript}\left(\phi_{i}\right.$ is a basis function of the $i^{t h}$ order). The basis functions are oscillatory in nature. The order of the basis function determines the frequency of its oscillations and its amplitude. Lower order basis functions will have higher amplitudes and lower frequencies whereas higher order basis functions will be vice versa. Basis functions of different orders are demonstrated in the figure below.

Basis functions of varying orders can be incorporated to the discrepancy function as shown in equation 28. When adding discrepancy to the model, the lower order basis function terms are added first, since these will have the most broad effect on the model output. After examining the effect of these basis functions on model output, higher order basis function terms may or may not be added. Since the higher order discrepancy terms will have lower amplitude and higher frequency, embedding these 

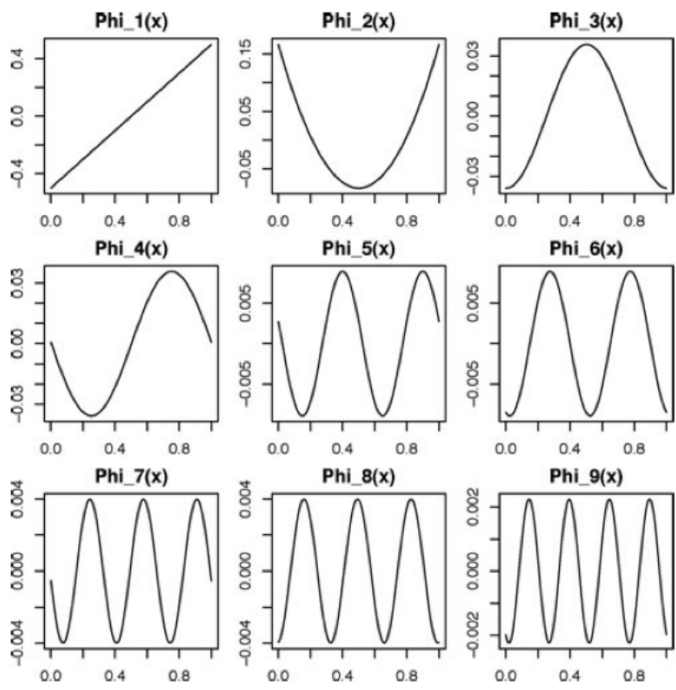

Figure 7: Basis Functions of Different Orders[42]

in the expression for discrepancy will enable the calibration process to fine-tune the model. They will also increase the number of mathematical operations needed for the discrepancy function, increasing the computation cost of the model.

As mentioned before, addition of discrepancy terms make the model more accurate at the cost of computational complexity. Since the primary goal of this work is to create a computationally inexpensive model, increased computational time of the model will only be acceptable upto a certain extent. Determining the optimum balance between model accuracy and computational complexity is a matter of trial and error. Bayesian Information Criterion(BIC) is used as the index to reflect the balance of computational complexity and model accuracy.

$$
\mathrm{BIC}=k(\ln (n))-2(\ln (L))
$$

where

$$
k=\text { no of parameters }
$$

$n=$ no of data points used for calibration

\section{$L=$ Maximum likelihood value for the model}

As more discrepancy terms are added to the model, the number of beta values increases, increasing the number of parameters of the model. Addition of discrepancy terms make the model more accurate, leading to higher likelihood $(L)$ values. These two processes impact the BIC value in two directions, with higher accuracy driving the $\mathrm{BIC}$ value lower and more complexity of the model driving it higher. Hence, the model that produces the minimum BIC values is considered to be the most efficient model. 


\subsection{Bayesian Calibration}

The final step in creating an accurate ROM is model calibration which essentially gives an estimation of the the parameter values in the model. This involves running the model for different parameter sets and comparing the results with the experimental data to decide which parameter values provide the best match with data. Widely used methods of calibration for mathematical models include Least Squared Error and Maximum Likelihood Estimation.

In this work, Bayesian calibration is used to calibrate the ROM to experimental data. Bayesian methods provide a distribution for the parameter values and model predictions instead of definite 'correct' values. This prevents over-confident use of the model. Also, using Bayesian calibration enables repeated estimation of the parameter distribution when new data or information is obtained. The methodology of Bayesian calibration is derived from Baye's Theorem, which states

$$
P(A \mid B)=\frac{P(B \mid A) P(A)}{P(B)}
$$

Essentially, the theorem states that the probability of an event A given event B, $\mathrm{P}(\mathrm{A} \mid \mathrm{B})$, is equal to probability of $\mathrm{B}$ given $\mathrm{A}, \mathrm{P}(\mathrm{B} \mid \mathrm{A})$, multiplied by probability of $\mathrm{A}, \mathrm{P}(\mathrm{A})$, and then divided by probability of $\mathrm{B}, \mathrm{P}(\mathrm{B})$. This information is simply extended to the case of mathematical models for Bayesican calibration. Event $\mathrm{A}$ is defined as an arbitrary parameter set $\theta$ (usually a vector) and event $\mathrm{B}$ is the given experimental data set $Z$. Therefore, the conditional probability $\mathrm{P}(\mathrm{A} \mid \mathrm{B})$ or from here on $\mathrm{P}(\theta \mid \mathrm{Z})$, is defined as the probability of a particular parameter set being true, given that the specific data set $\mathrm{Z}$ is observed. In any multidimensional parameter space, this conditional probability has an unknown distribution and is referred to as the Posterior distribution $(\pi)$. Obtaining a posterior distribution is the goal of the Bayesian calibration. Furthermore, the conditional probability $\mathrm{P}(\mathrm{Z} \mid \theta)$ is the probability that the experimental data set $\mathrm{Z}$ is observed given that the parameter set $\theta$ is true for the model. This term is also known as the Likelihood $(L)$. Next, $\mathrm{P}(\theta)$, which is the simply the probability of any parameter set being observed from the entire parameter space, is referred to as the Prior probability and it represents belief of a certain parameter set being true prior to any information from data being taken into account. This prior is considered to be uniform if no prior information is known about the parameters, since all values of the parameters within the bounds are equally likely before calibration. Finally, the term $\mathrm{P}(\mathrm{Z})$ can be expressed using the Law of Total Probability for a continuous random vector and it is computed as the following integral:

$$
P(Z)=\int_{\theta} P(Z \mid \theta) P(\theta) d \theta
$$

The above integral, also known as the marginal likelihood, is often very difficult to evaluate analytically, especially if there are many parameters (high-dimensional 
integration). However, the probability is only dependent on the observed data $\mathrm{Z}$ and is the same for every possible parameter set $\theta$ if a uniform prior is used. Thus, Bayes' theorem (refer eqn here) can be used to obtain the Posterior distribution, with the integral being only a normalizing factor or constant, thereby giving a proportional relation as follows:

$$
P(\theta \mid Z) \propto P(Z \mid \theta) P(\theta)
$$

The above generic form can now be extended to this work. Expressing the Posterior distribution function as $\Pi$, the Likelihood function as $L$, the prior distribution function as $\Omega$, the discrepancy parameters as $\beta$ and including the observational error variance $(\psi)$ as an additional unknown parameter for the calibration, we get the following useful expression:

$$
\Pi(\theta, \beta, \psi \mid Z) \propto L(Z \mid \theta, \beta, \psi) \Omega(\theta, \beta, \psi)
$$

The likelihood, $L(Z \mid \theta, \beta, \psi)$ is the probability of observing the data-set $\mathrm{Z}$ for the given set of parameters. $\Pi(\theta, \beta, \psi \mid Z)$ is the posterior distribution function and $\Omega(\theta, \beta, \psi)$ is the prior distribution function. As it can be seen, the posterior distribution is dependent on the prior distribution. This is what enables Bayesian calibration to take into account prior beliefs about the parameters. $\theta, \beta$ and $\psi$ will have uniform distributions if nothing is known beforehand about the parameter distribution, since all values of the parameters will be equally likely then. However, if prior knowledge is available, the prior distribution will affect the value of $\Omega(\theta, \beta, \psi)$ enabling the posterior distribution to vary accordingly as well.

In the case of this work, the prior for discrepancy parameters $\beta$ was a multivariate normal distribution. $\theta$ parameters were modeled as having normal distribution priors with lower and upper bounds. This is because they are physical parameters and will have values within a given reasonable range obtained by previous experiments. The following bounds were used for $\theta$ parameters. 


\begin{tabular}{||cccc||}
\hline Parameter & Upper Limit & Lower Limit & SDEV \\
\hline \hline$\Delta \mathrm{H}_{1}(J / k g)$ & 200000 & -100000 & 100 \\
\hline$\Delta \mathrm{H}_{2}$ & 200000 & -100000 & 100 \\
\hline$\Delta \mathrm{H}_{3}$ & 200000 & -100000 & 100 \\
\hline$\Delta \mathrm{S}_{1}(J / K)$ & 200 & -200 & 19 \\
\hline$\Delta \mathrm{S}_{2}$ & 200 & -200 & 19 \\
\hline$\Delta \mathrm{S}_{3}$ & 200 & -200 & 19 \\
\hline
\end{tabular}

Table 2: Parameter Bounds

\begin{tabular}{||cccc||}
\hline Parameter & Upper Limit $\left(10^{x}\right)$ & Lower Limit $\left(10^{x}\right)$ & $\operatorname{SDEV}\left(10^{x}\right)$ \\
\hline \hline$A_{1}$ & 3.954 & 0 & 2.279 \\
\hline$A_{3}$ & 3.954 & 0 & 2.279 \\
\hline
\end{tabular}

Table 3: Parameter Bounds With Log-scale Sampling

The above bounds were found using approximation from literature depicting similar processes occurring in similar conditions. The bounds for $\beta$ parameters were found using trial and error, since these are parameters for the statistical portion of the model and hence has no definite limit. Large values of $\beta$ parameters were avoided since the exponent $(e)$ of the discrepancy function value is used to update the parameters $\left(\Delta \mathrm{S}_{1}=\Delta S_{1}\right.$. $\left.\exp (\delta)\right)$. Large values of $\beta$ will lead to unrealistically large values of the parameters or even over-flow of program variables. The pre-exponential factors $A_{1}$ and $A_{3}$ for the rate constants were explored using log-scale during calibration.

It is difficult to obtain an analytic expression for posterior distribution from the relation described in equation 25. As a result, Markov Chain Monte Carlo (MCMC) sampling is used to sample points from the posterior distribution and create an approximate distribution.

A Markov Chain or Markov Process is a stochastic model describing a sequence of possible events in which the probability of each event depends only on the state obtained in the previous event. A process satisfies the Markov property if one can make predictions for the future of the process based solely on its present state just as well as one could knowing the process's full history.

On the other hand, very generally speaking, Monte-Carlo is the practice of estimating the properties of a distribution by examining random samples from the distribution. The two analysis techniques are combined to form Markov Chain Monte Carlo (MCMC) method of sampling distributions.

MCMC is a computer-driven sampling method. It allows one to characterize a distribution without knowing all of the distribution's mathematical properties by randomly sampling values out of the distribution. A particular advantage of using MCMC is that it can be used to draw samples from distributions even when all that is known about the distribution is how to calculate the density for different samples. For 
example, instead of finding the mean of a normal distribution by directly calculating it from the distribution's equations, a Monte-Carlo approach would be to draw a large number of random samples from a normal distribution, and calculate the sample mean of those. Calculating the mean of a large sample of numbers can be much easier than calculating the mean directly from the normal distribution's equations especially in cases where random samples are easy to draw but the distribution's equations are hard to work with in other ways. The Markov chain property of MCMC is the idea that the random samples are generated by a special sequential process. Each random sample is used as a starting point to generate the next random sample (hence the chain). A special property of the chain is that, while each new sample depends on the one before it, new samples do not depend on any samples before the previous one (hence the "Markov" property).

For the purpose of this work, the MCMC routine initially draws samples from the prior distribution. The likelihood corresponding to the specific parameter set is then determined. The value of the likelihood of the current parameters set is compared to the the likelihood value of the immediate previous sample of parameter set. This comparison is used to determine the probability the proposed sample is accepted and included in the posterior distribution. Based on this probability, the draw is either accepted and becomes the next base point for the random walk of the MCMC, or the draw is rejected and a new draw is taken. Hence, any subsequent draw from the sample will only depend on the draw immediately before it, giving the process its 'Markov Property'. As the number of MCMC steps taken increases, the random walk through parameter space eventually starts to show a pattern. This finally converges on a confined region, defined the posterior distribution, and finalizes the ROM. The parameter values in this posterior region are used to make model predictions. 


\subsection{Calibration Data}

The data for calibration is obtained from the publication of $\mathrm{Xu}$ and Froment[15]. Below is a plot used for data collection from their work.

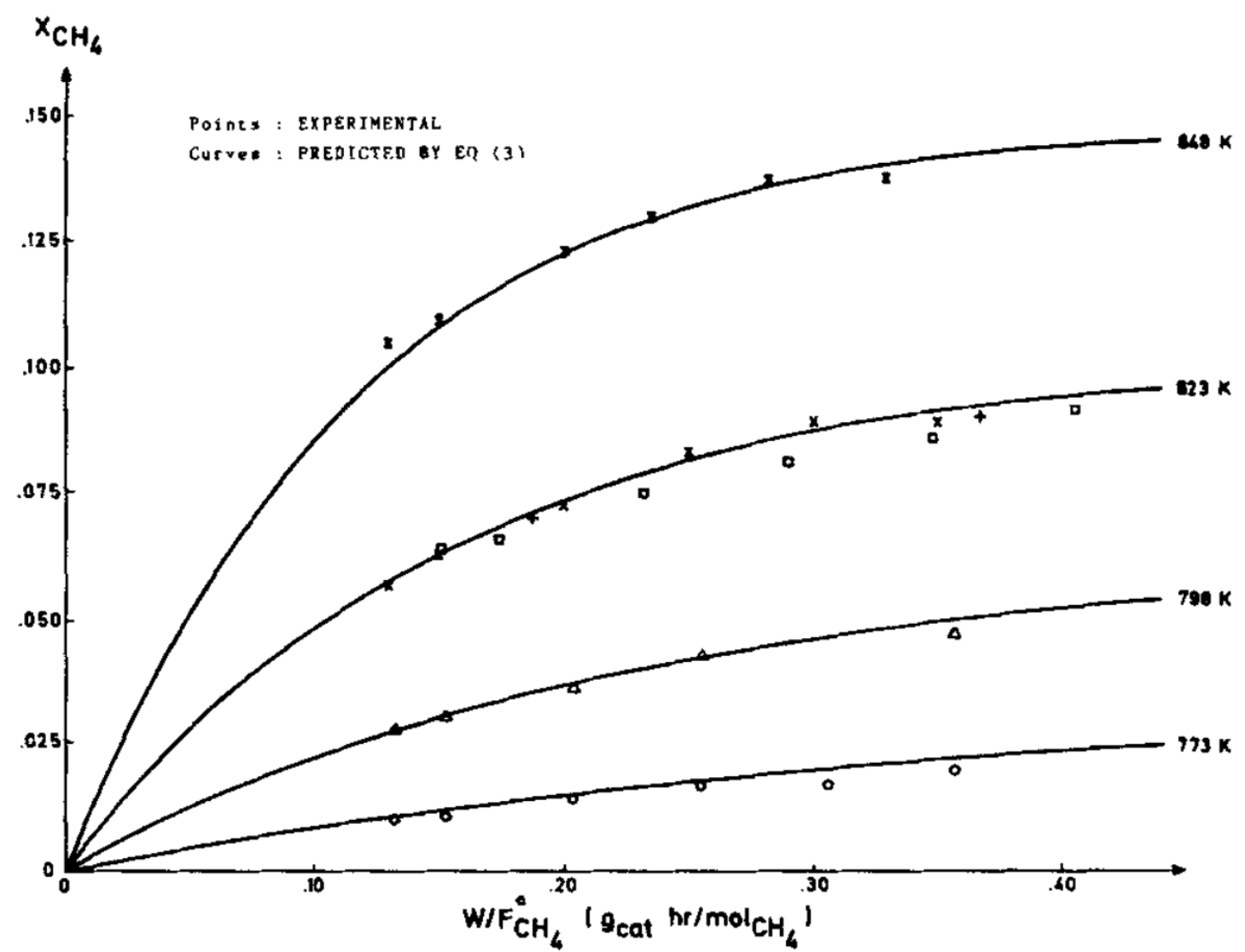

Figure 8: Example Calibration Data[15]

All the points on the plot represent the steady-state of the system for different configurations. On the x-axis are values of methane inflow given in the form of space time, where

$$
\begin{gathered}
W=\text { mass of catalyst in grams }=0.4 \mathrm{~g} \\
F_{C H_{4}}^{O}=\text { methane inflow in mole } / \mathrm{hr}
\end{gathered}
$$

Using the value of $W$, x-values of the plots are then converted to methane inflow values in $\mathrm{mol} / \mathrm{s}$.

On the $y$-axis is the conversion fraction of methane, whose formula is:

$$
Y_{\mathrm{CH}_{4}}=\frac{\mathrm{CH}_{4} \text { Consumed }}{\mathrm{CH}_{4} \text { Inflow }}=\frac{\mathrm{CH}_{4} \text { Inflow }-\mathrm{CH}_{4} \text { Outflow }}{\mathrm{CH}_{4} \text { Inflow }}
$$

$\mathrm{CH}_{4}$ inflow is the constant feed value of methane for a particular configuration. $\mathrm{CH}_{4}$ outflow is calculated using the expression for total outflow and the pressure 
fraction for methane.

$$
\mathrm{CH}_{4} \text { Outflow }=\frac{P_{C H 4}}{P_{\text {Total }}}\left(N_{\text {TOTAL }}+\left(2 V r_{1}+3 V r_{3}\right)\right)
$$

The system is isothermal and the different plots correspond to different temperatures of the system at different configurations. Points from these plots are extracted using the MATLAB library GRABIT[43]. Grabbing the points in this automated manner ensure that data points read from plot are accurate up to a single pixel. Calibration is carried out using data for temperatures at $848 \mathrm{~K}, 823 \mathrm{~K}$ and $773 \mathrm{~K}$. The data corresponding to the temperature at $798 \mathrm{~K}$ is left out to test the interpolation capabilities of the model.

\subsubsection{CSTR Assumption}

The equipment used in literature is a tank reactor with inflow and outflow gases continuously entering and exiting. Keeping the dimension of the reactor intact, the

reactor is assumed to be a Continously Stirred Tank Reactor(CSTR) for the purposes of this work. 


\section{Calibration and Results}

As mentioned earlier, the discrepancy terms are added incrementally to the reduced model. Initially a calibration of the reduced order model with no discrepancy and only the physical parameters is carried out. This gives an initial posterior distribution of the parameters. The most likely values of the parameters from this distribution are chosen as the starting point for the next calibration. This enables the calibration routine to avoid repeated burn-ins. Burn-ins are regions of the parameter space that is traversed by the MCMC routine while it searches for the true parameter space. Starting subsequent calibrations from the best region of previous calibration ensures that the MCMC routine is closer to the desired distribution and hence makes convergence of the calibration faster. After the initial calibration, discrepancy terms are added to the model. The new model is subsequently calibrated to find the new posterior distribution of parameters. After each calibration, the model prediction is compared to the data and the Bayesian Information Criterion(BIC) value corresponding to the best model from the calibration is determined. This process is carried out iteratively till a minimum BIC value model is found. This is the most optimized model, with the highest accuracy for the lowest possible computational complexity.

During the course of the calibration, one of the problems faced initially was solver error or solver failure. Relatively large bounds are used for all parameters initially, since there is little information known about them. As a result, during the course of calibration, some combinations of parameter values lead to a system of ODEs that the solver fails to solve. This may be because the ODEINT solver fails to converge and actually diverges, leading to contracting time steps which eventually exceeds the maximum number of iterations. Another possible reason could have been a overly low error tolerance, leading to very small time steps and hence massive number of iterations. To preclude this source of error, relative error tolerance levels were kept at the optimum value of $10^{-3}$. The experimental data obtained from plots in literature could be roughly considered to be accurate up to the second decimal place $\left(10^{-2}\right)$ so this tolerance is more than enough for calibration purposes.

Tweaking the error tolerance reduced solver failure considerably but did not completely omit it. So, to ensure the calibration routine avoids regions of the parameter space where solver failure is likely, a high error value is returned by the model whenever the solver fails. This keeps the Bayesian calibration routine reasonably away from solver failure regions. 


\subsection{Calibration Plots}

The final optimized model is derived using a step-by-step process, with calibration, model evaluation and discrepancy addition at each step. The general methodology is described by the following flowchart.

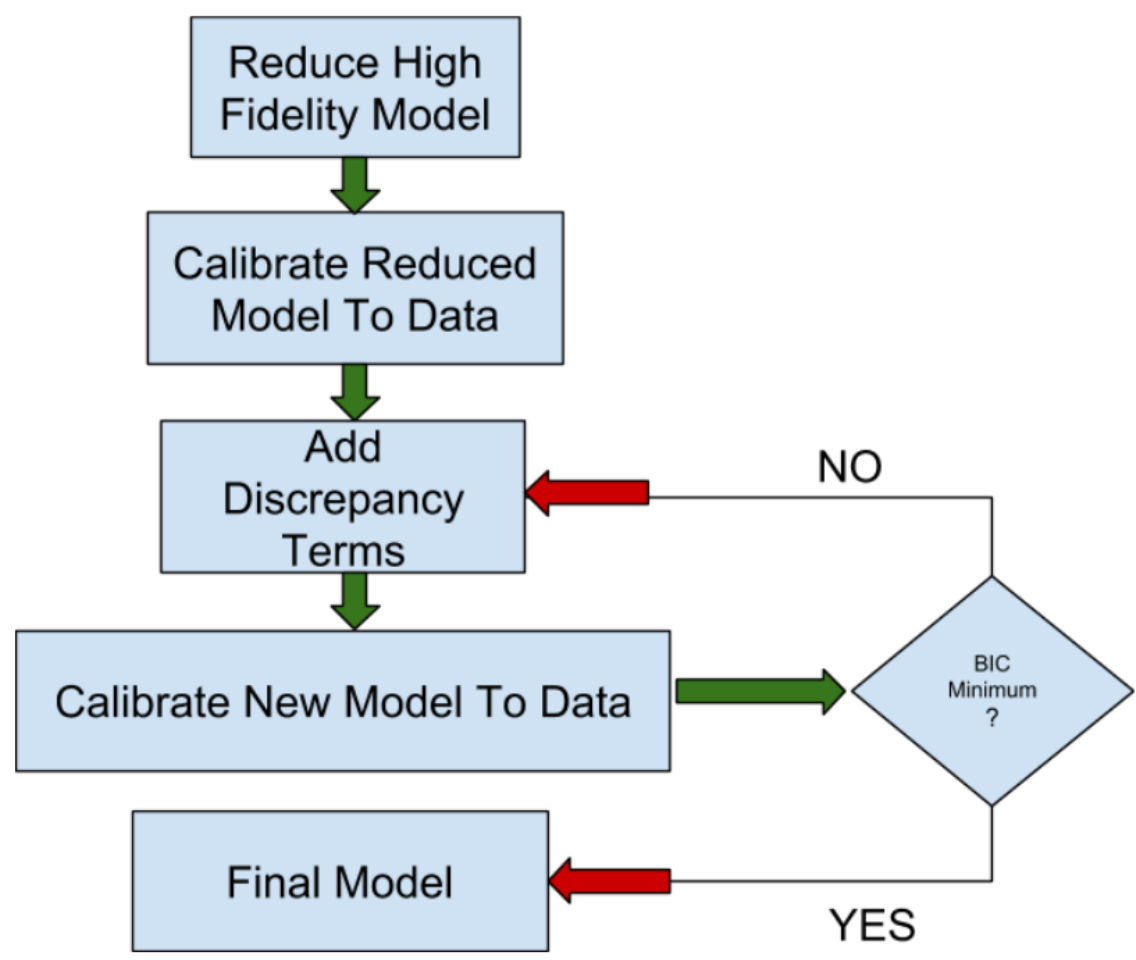

Figure 9: Methodology

Initially, after addition of each new discrepancy term, the BIC value would decrease. However, after a certain number of terms, there would be a point where BIC starts increasing again. This turning point can be be considered the minimum BIC value since the plot of BIC against number of discrepancy terms added will have a convex shape in general.

The posterior distribution of parameters obtained from calibration are visualized using scatter plots. The extent to which the model agrees with the experimental data is observed by model prediction plots. The evolution of the posterior distribution of parameters during calibration is observed using parameters estimation plots.

At first the reduced model without any discrepancy is calibrated to data. This results in the following group of scatter plots. 

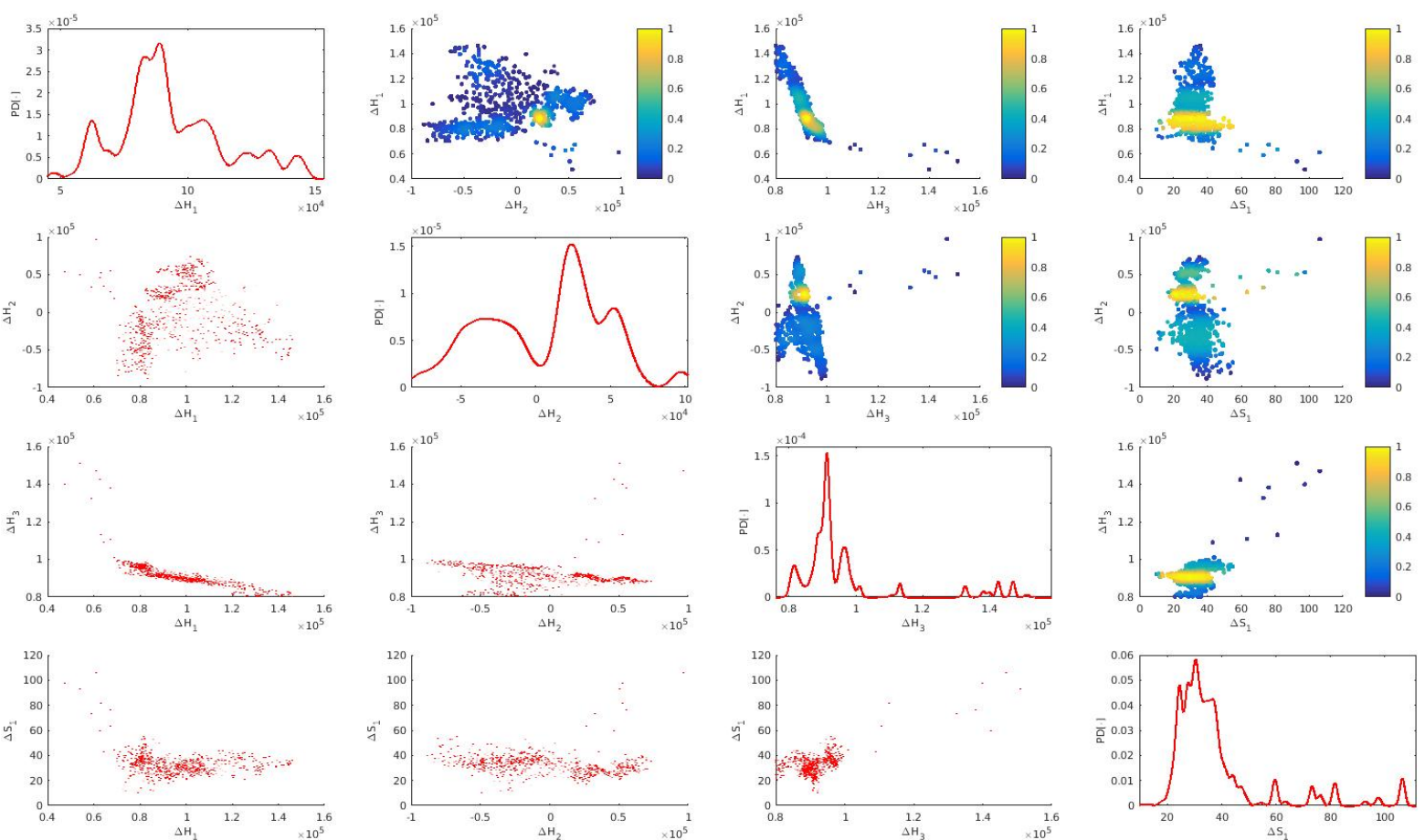

Figure 10: Scatter Plot - Physical Parameters

The scatter plots consist of a collection of plots which have a parameter on the $\mathrm{x}$ axis and another on y axis. Together, these plots give an idea about the distribution of all the parameters in the multi-dimensional parameter space. In the figure above the plots along the diagonal are parameter values vs probability density plots. Plots on lower and upper half triangle plots are scatter plots. The upper half triangle plots contain color-based representations of the parameter distribution. A higher density of points mean the region will be more yellow and a lower density means the region will be more blue.

As it can be seen from the figure, certain parameters show a more definite posterior distribution than others. This is due to the limited amount of calibration data available. As mentioned earlier, the steady-state total conversion of methane and carbon dioxide was the only data available from literature. This left out three other species which would take part in the proposed reduced reaction network. Hence, all parameters will not have a sizable impact on the cost function for the calibration, since only two of the five model outputs are considered. The parameters that do have an impact, such as $\Delta H_{1}$ and $\Delta H_{3}$ do show a strong dense distribution, as can be seen from from the scatter plots and color coded distributions.

The entire distribution of physical parameters can be described using a group of scatter plots. This distribution holds significance because the values of physical parameters will remain in this range for similar operating conditions. The distribution of physical parameters is illustrated in the next figure. 


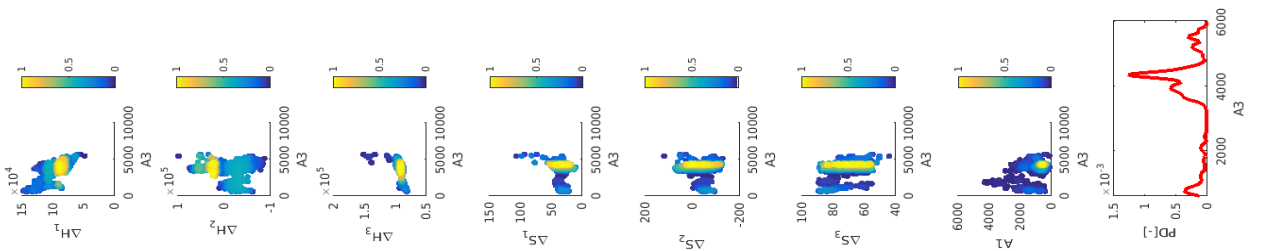

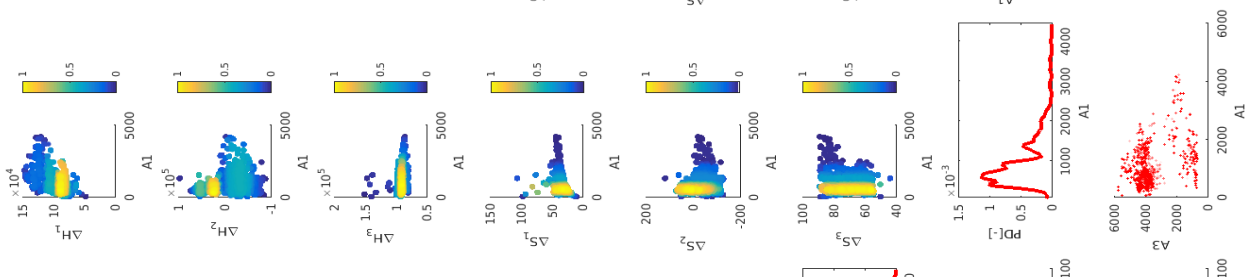

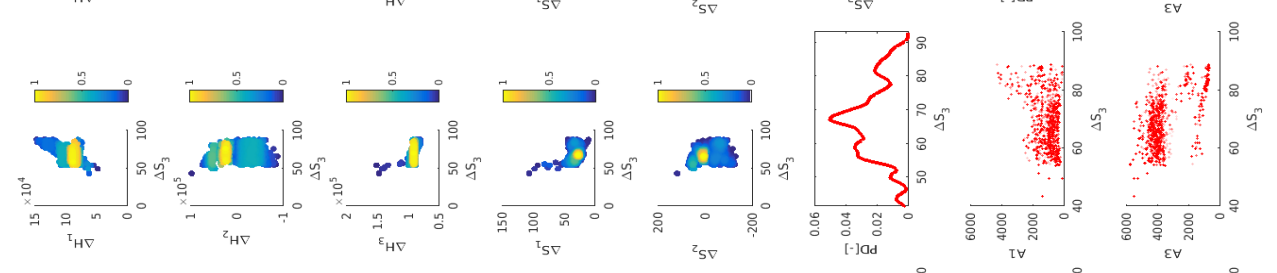

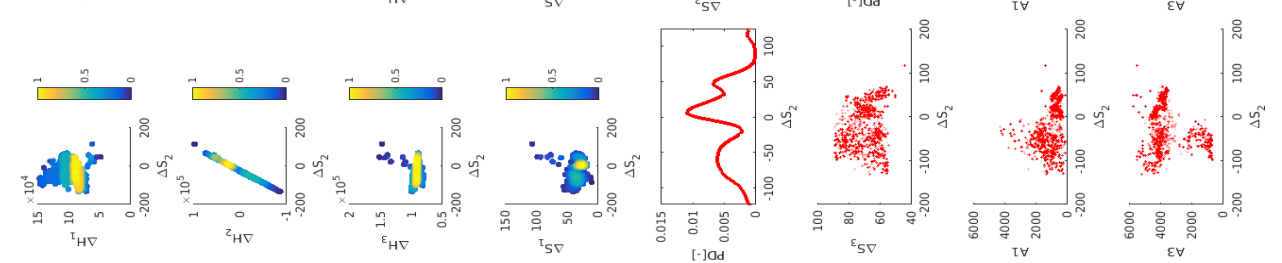

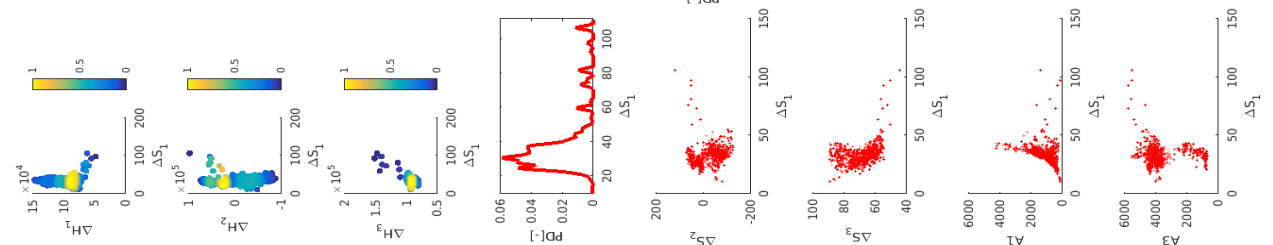

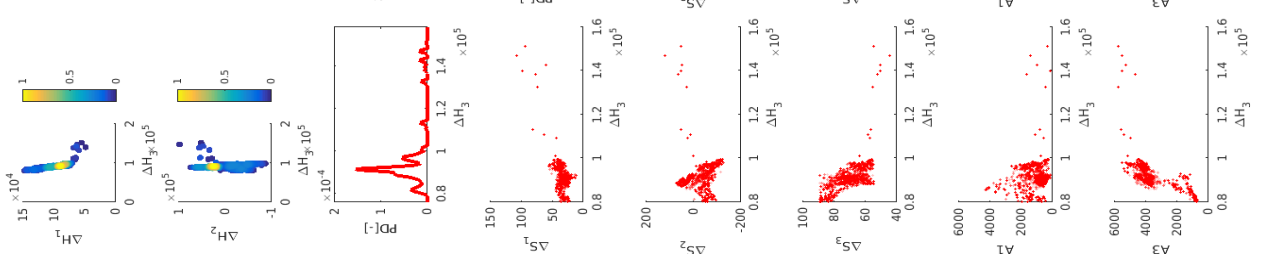

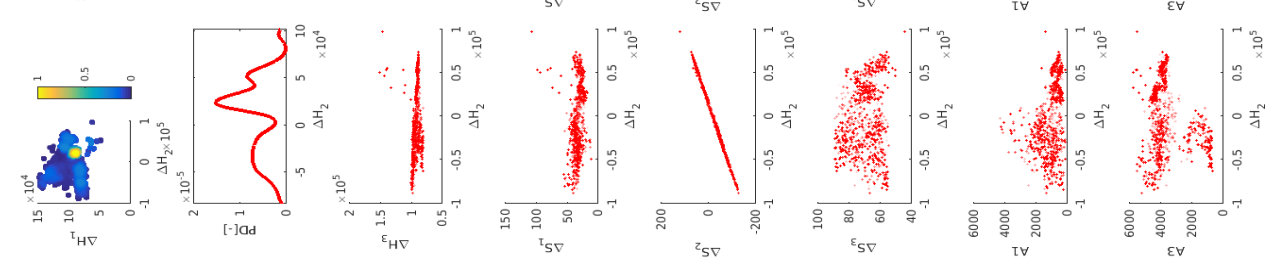

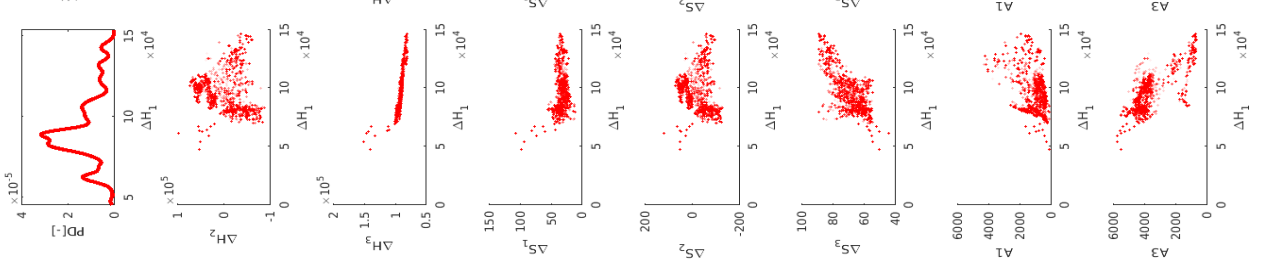

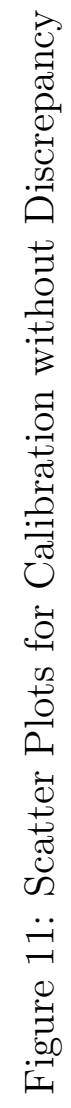


The trajectory of the MCMC routine when traversing this 8-dimensional parameter space is observed using parameter estimation plots as illustrated next.
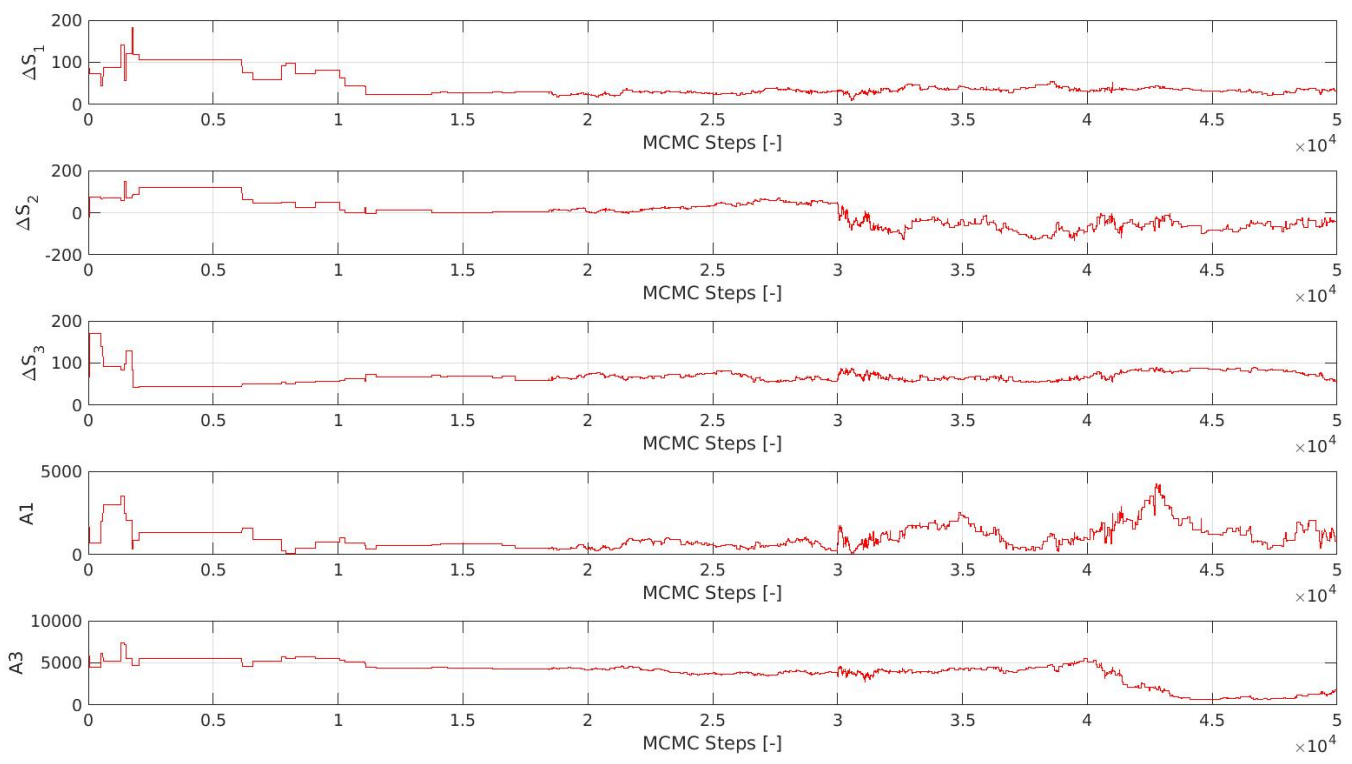

Figure 12: Parameter Estimation Plots without Discrepancy I

The purpose of the parameter estimation plots is to observe any patterns of interest that arise from the calibration process. As far as physical parameters and results are concerned, parameter estimation plots hold little significance. However, it helps with the identification of errors or other issues with the calibration process by enabling one to observe the movement of the MCMC routine during calibration. For instance, if, due to a bug in the code, one of the parameters is not explored thoroughly during calibration, parameter estimation plots will identify the said parameter. It can also assist in sensitivity analysis. Parameters that do not affect the Quantity of Interest(QoI) appreciably will show very little movement in the plots. 

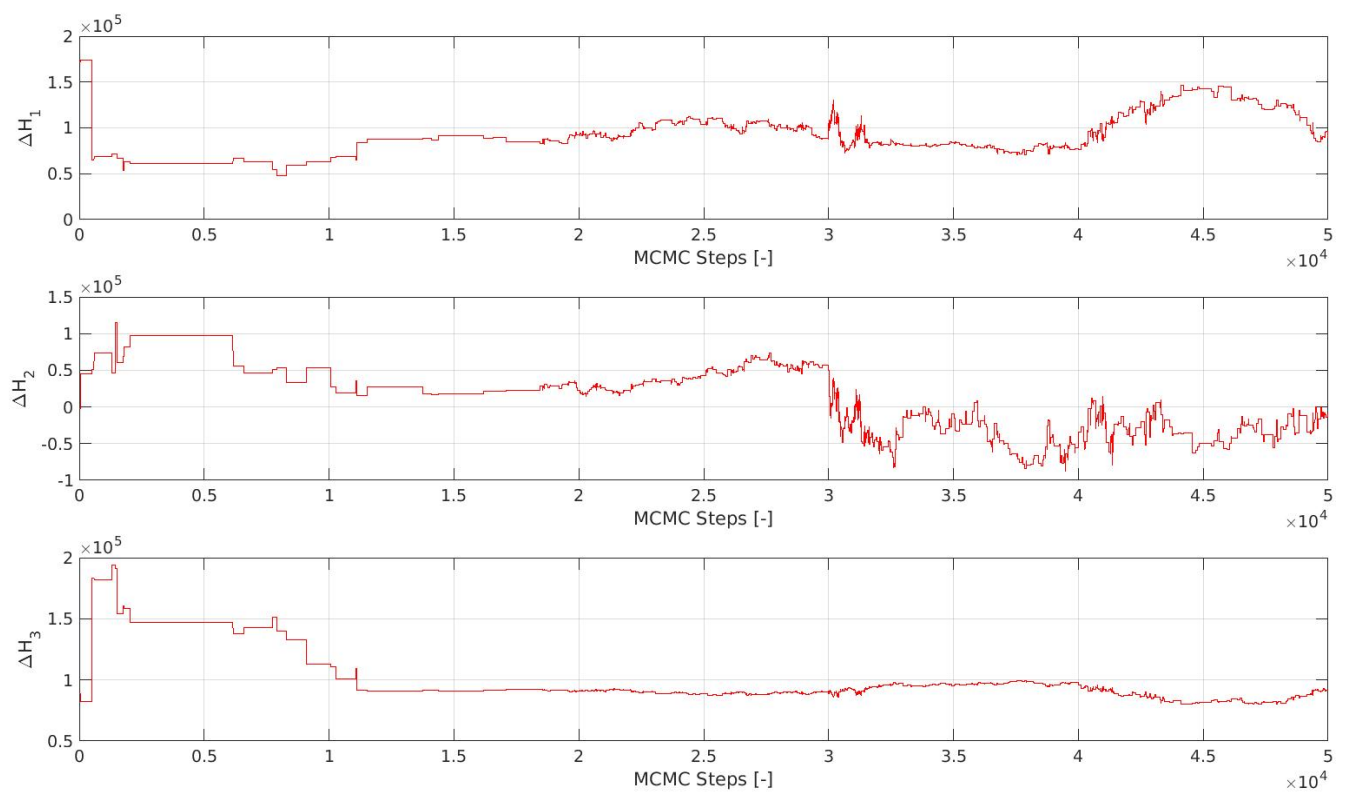

Figure 13: Parameter Estimation Plots without Discrepancy II

Following the parameter estimation plots, model predictions are made with the calibrated model without discrepancy, as illustrated in the next figure. 

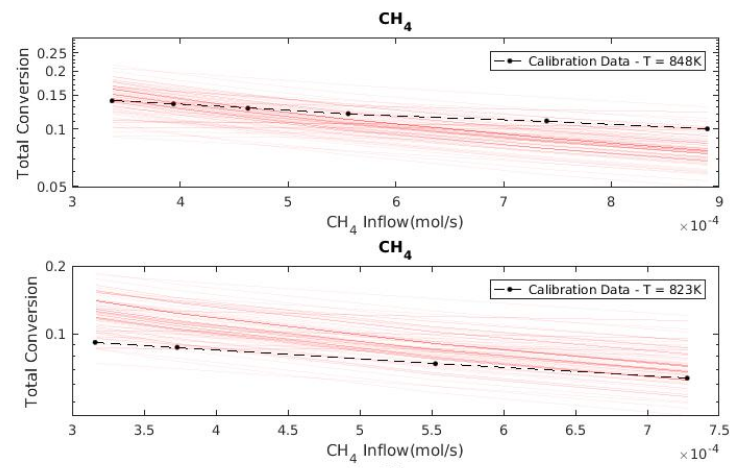

$\mathrm{CH}_{4}$

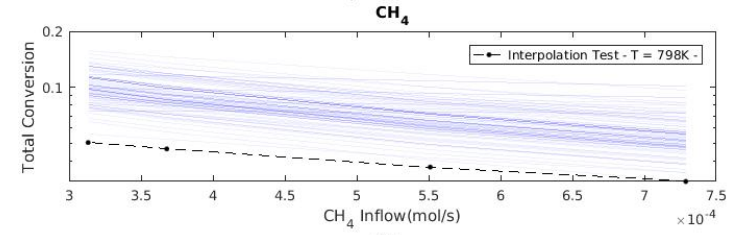

$\mathrm{CH}_{4}$

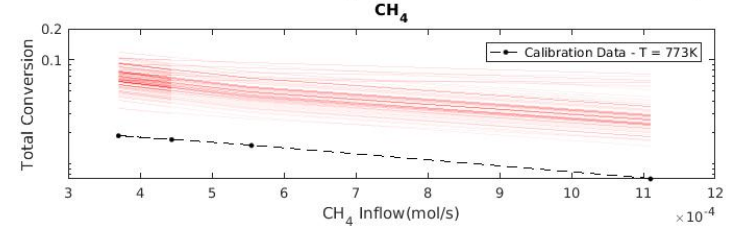

$\mathrm{CO}_{2}$

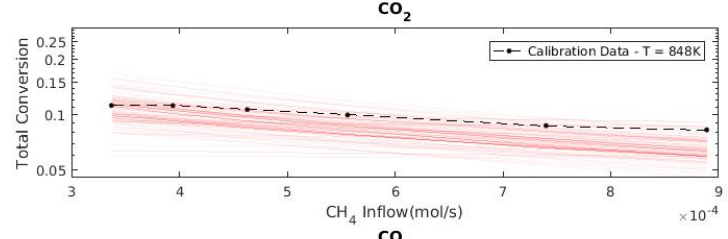

$\mathrm{CO}_{2}$

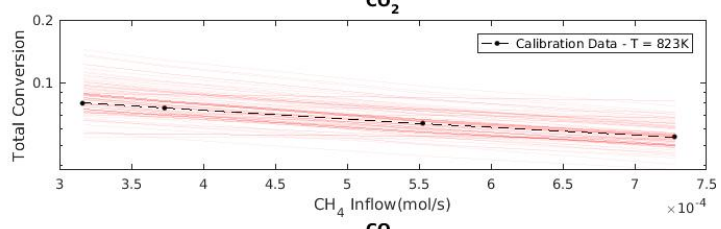

$\mathrm{CO}_{2}$

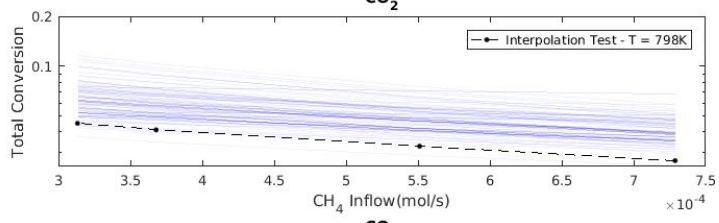

$\mathrm{CO}_{2}$

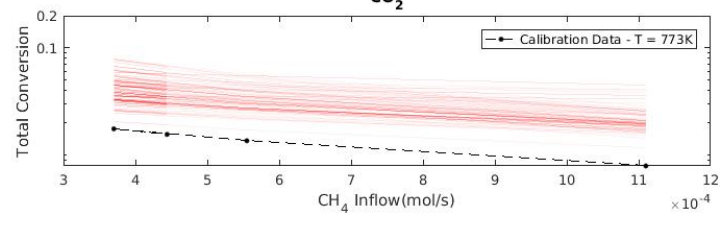

Figure 14: Model Prediction without Discrepancy

In the given figure, patches of thin colored lines are used to represent the model results. This is consistent with the process of Bayesian Calibration, because Bayesian Calibration is supposed to produce a set of most probable models rather than a single supposedly perfect model. The variation in results of the final models produced by calibration after convergence indicate the uncertainty associated with the given model. Hence, a thick patch, corresponding to a final set of models with significantly varying results, means there is high degree of uncertainty.

The red patches correspond to the data used for calibration and the blue patches refer to data used to test the interpolation capabilities of the model. The experimental data in paper consisted of steady-state conversion fractions at temperatures $\mathrm{T}=$ $848 \mathrm{~K}, 823 \mathrm{~K}, 798 \mathrm{~K}$ and $773 \mathrm{~K}$. Only the data for $\mathrm{T}=848 \mathrm{~K}, 823 \mathrm{~K}$ and $773 \mathrm{~K}$ were used for calibration. The data corresponding to $\mathrm{T}=798 \mathrm{~K}$ were left out to test the interpolation capabilities of the presented methodology. As it can be seen, relatively large patches are observed for all the plots in general, implying a high degree of uncertainty. Also, there is no coverage for data corresponding to $\mathrm{T}=798 \mathrm{~K}$ and $\mathrm{T}=$ $773 \mathrm{~K}$. In general, this is not a very good model. This is of course expected, since a lot of reductions were made to the original reaction network. Discrepancy, which is supposed to act as a low-computation-cost substitute for the omitted elements, has not been introduced yet.

After this initial calibration without discrepancy, discrepancy terms are added incrementally, to find the optimum model. In the initial set of calibrations, the main 
effects are included one by one. At first a single main effects with a $\beta$ parameter is added with the form of the discrepancy function being the following.

$$
\delta=\left(\beta_{1,1}\right) \phi_{1}\left(x_{1}\right)
$$

Only basis functions of order one $\left(\phi_{1}\right)$ are used for this discrepancy function, meaning that it will have some broad effects on the model output. This produces the model prediction illustrated in the next figure.
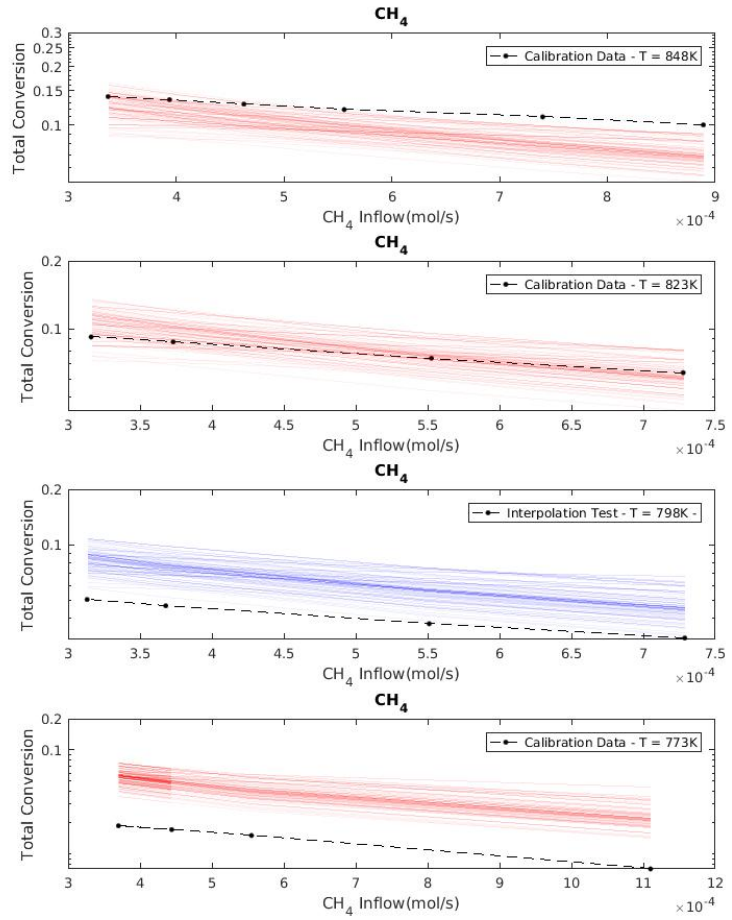
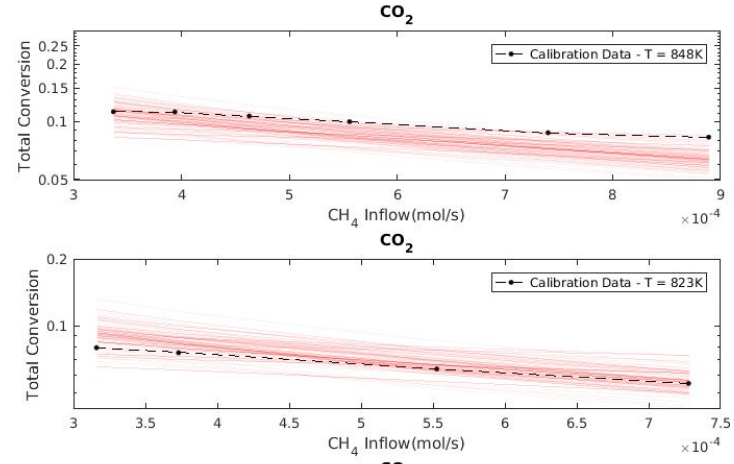

$\mathrm{CO}_{2}$

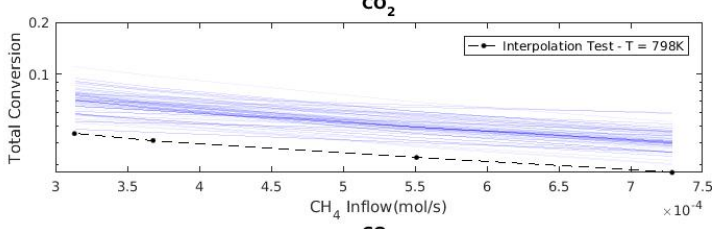

$\mathrm{CO}_{2}$

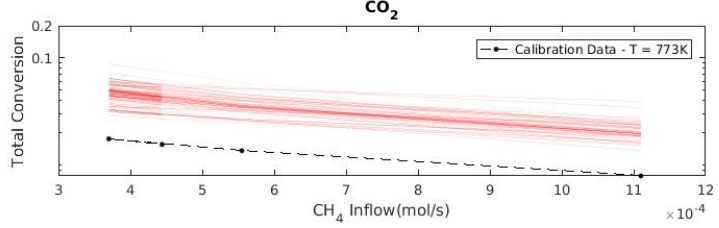

Figure 15: Model Prediction With Single Beta Discrepancy Function

Introducing this sparse discrepancy function does not visibly improve the coverage of the experimental data in general. The data corresponding to $\mathrm{T}=773 \mathrm{~K}$ still remains mostly uncovered. Interestingly, a better coverage is observed for the interpolation test data than for calibration data corresponding to $\mathrm{T}=773 \mathrm{~K}$. A possible reason for this could be that $\mathrm{T}=798 \mathrm{~K}$ is closer to the first two calibration data sets $\mathrm{T}=848 \mathrm{~K}$ and $823 \mathrm{~K}$. Trying to cover the experimental data set for $\mathrm{T}=773 \mathrm{~K}$ simply moved the model away from covering $\mathrm{T}=848 \mathrm{~K}$ and $823 \mathrm{~K}$, which would increase the cost function of the calibration and cause it to relapse back to the previous region.

The major flaw in the plot above is the fact that the patches/bands are still very wide, representing a large amount of uncertainty regarding the model. So, clearly more parameters will need to be added to improve the model.

Finally, all main effects are included in the discrepancy function, as demonstrated in equations 17. 
Each main effect has basis functions of order 1 and 2 .

$$
\sum_{i}\left(\beta_{C H_{4}, i}\right) \phi_{i}\left(P_{C H_{4}}\right)=\left(\beta_{C H_{4}, 1}\right) \phi_{1}\left(P_{C H_{4}}\right)+\left(\beta_{C H_{4}, 2}\right) \phi_{2}\left(P_{C H_{4}}\right)
$$

This produces a definite distribution for the $\beta$ parameters.
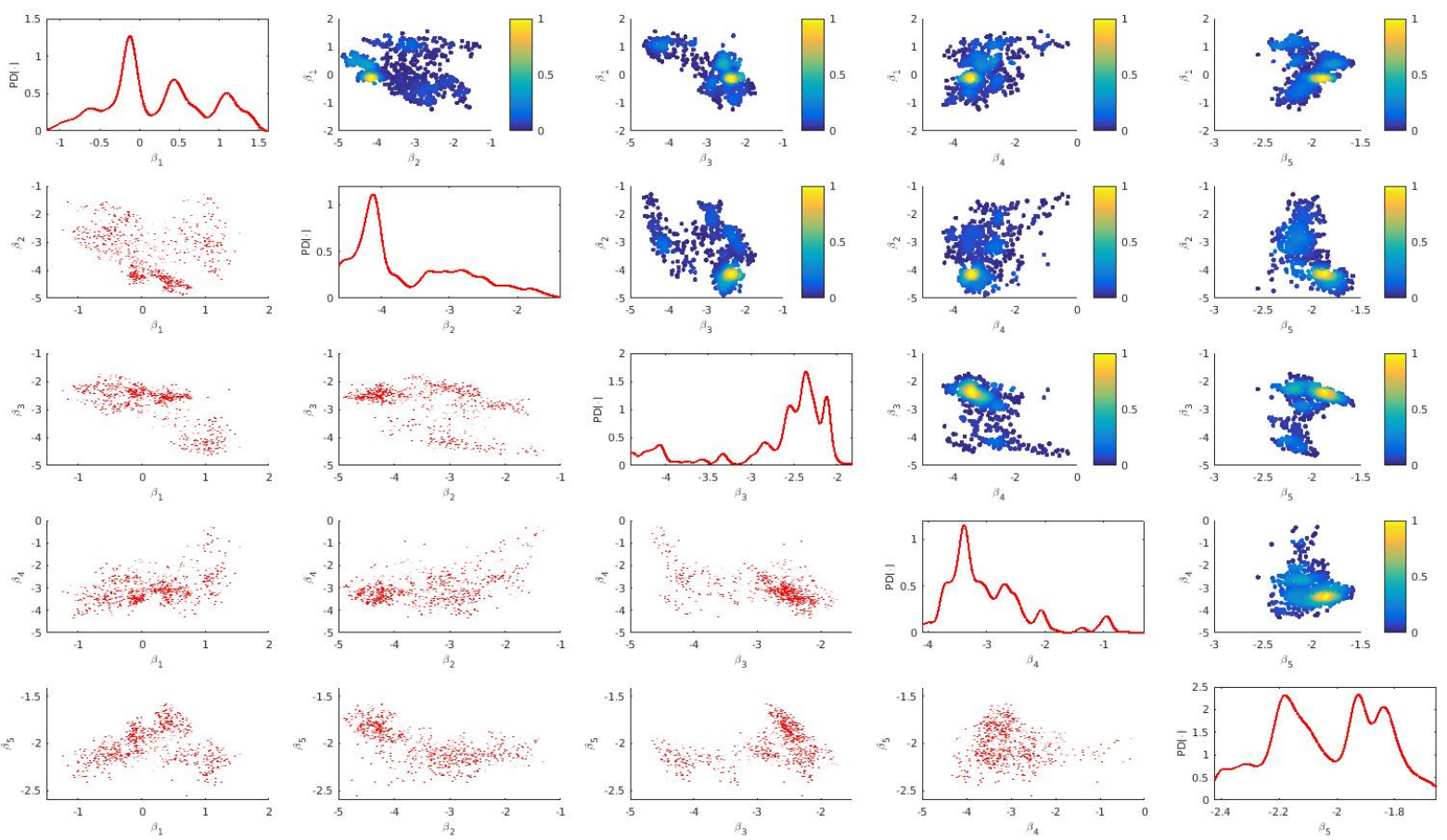

Figure 16: Distribution of Sample Beta Parameters for Main Effects

The following parameter estimation plots for the $\beta$ parameters and model prediction plots are observed for this particular calibration. 

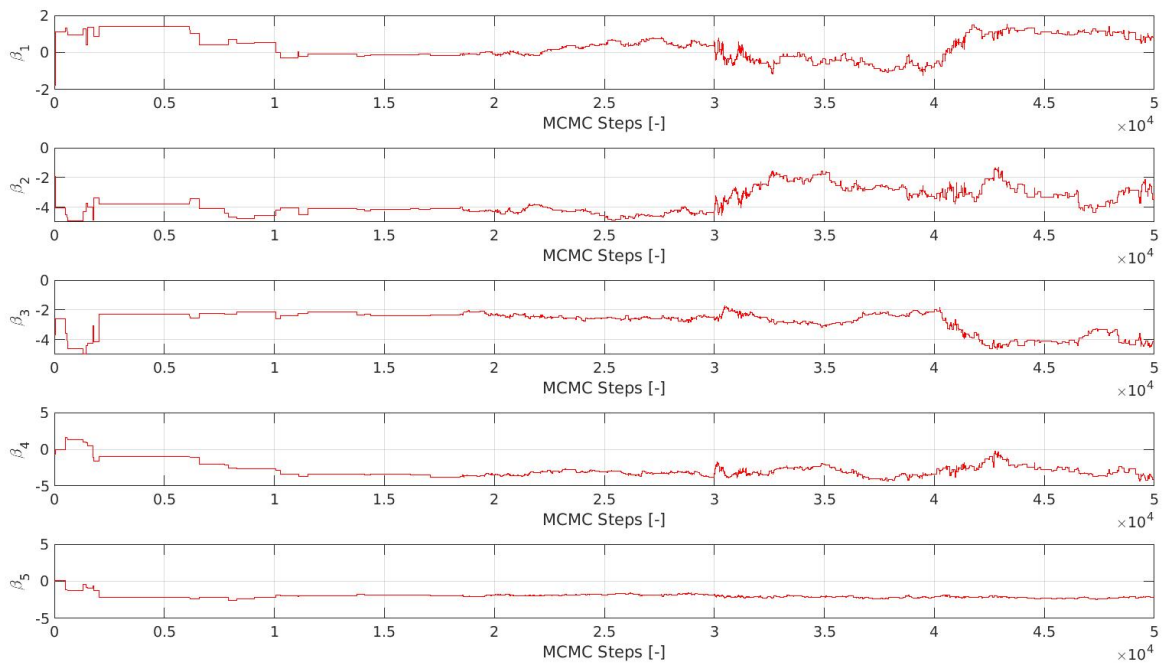

Figure 17: Beta Parameters Estimation Plots for Main Effects Calibration

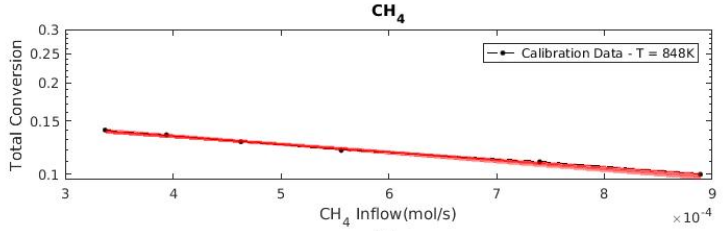

$\mathrm{CH}_{4}$

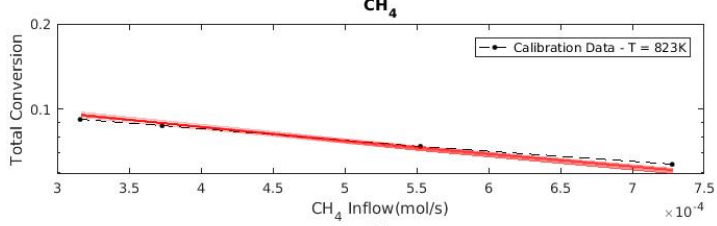

$\mathrm{CH}_{4}$

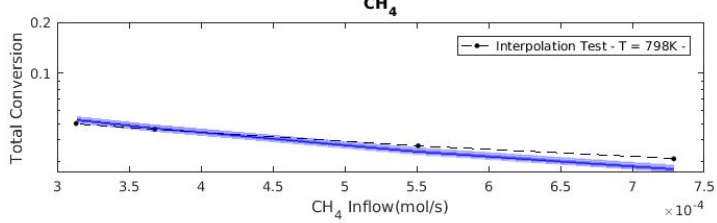

$\mathrm{CH}_{4}$

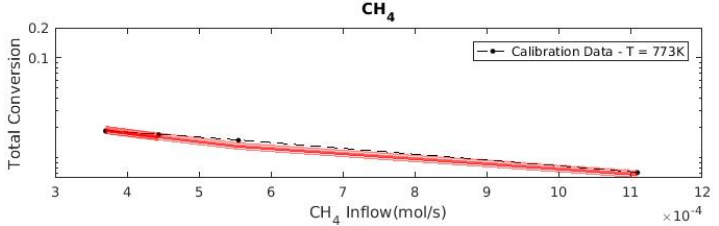

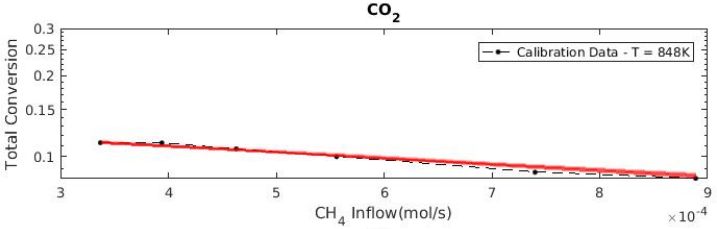

$\mathrm{CO}_{2}$

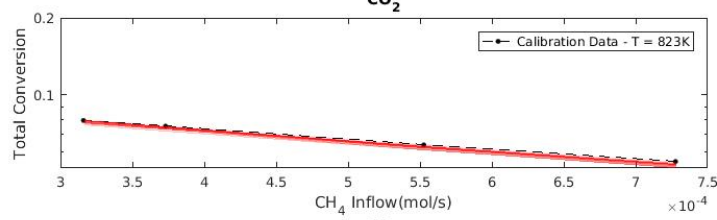

$\mathrm{CO}_{2}$

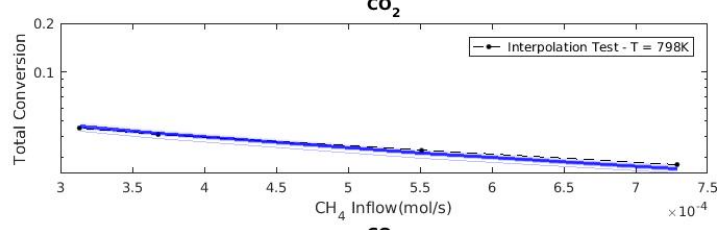

$\mathrm{CO}_{2}$

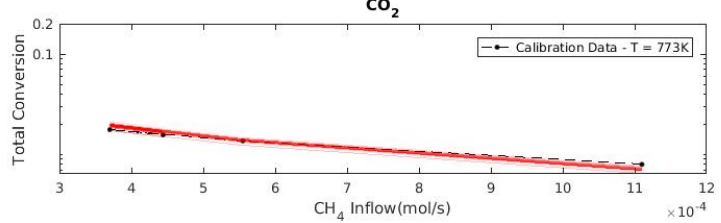

Figure 18: Prediction for Model With All Main Effects Incorporated

As it can be seen from the model prediction above, some regions of experimental data are not covered by this model. However, much thinner bands are observed, meaning significant reduction in the amount of uncertainty in the model. 
More discrepancy terms can be added to the model either by increasing the order of basis functions or by introducing higher order interactions. There is no definite advantage in choosing one over the other, which makes the addition of discrepancy somewhat of a trial and error process. An examination of the high fidelity model to find out which specific interaction the discrepancy terms are representing may enable one to make a more educated decision. However, that was beyond the scope of this work.

Adding more terms to the discrepancy function in this manner will yield better and better coverage of the experimental data with thinner and thinner bands. However, as discussed before, this will also continue to increase the computational cost of the model. Clearly, a balance needs to be reached between model prediction accuracy/certainty and the computational cost of the model. To know when this balance is reached, there needs to be an index that indicates the level of optimization of the model(i.e how streamlined the model is when both accuracy and computation cost are considered). As stated before, Bayesian Information Criterion, from equation 21, is used for this purpose in this work.

$$
\mathrm{BIC}=k(\ln (n))-2(\ln (L))
$$

Here $\mathrm{k}$, the total number of parameters, takes into account the computational cost of the model and $L$, the maximum likelihood, considers the accuracy of the model. The total number of data points used for calibration,n, is constant for all models in this work. Hence, increase in number of parameters, $\mathrm{k}$, will increase BIC whereas increase in accuracy and therefore likelihood, $L$, will decrease BIC. Hence, BIC will represent the balance of these two factors. The model with the minimum value of BIC can be considered the optimum model.

To find the optimum model in this manner, a (BIC vs Model No) plot is constructed. Each point on this plot refers to a model with a certain structure of the discrepancy function and its corresponding BIC value. As the model number increases, the number of parameters increases.

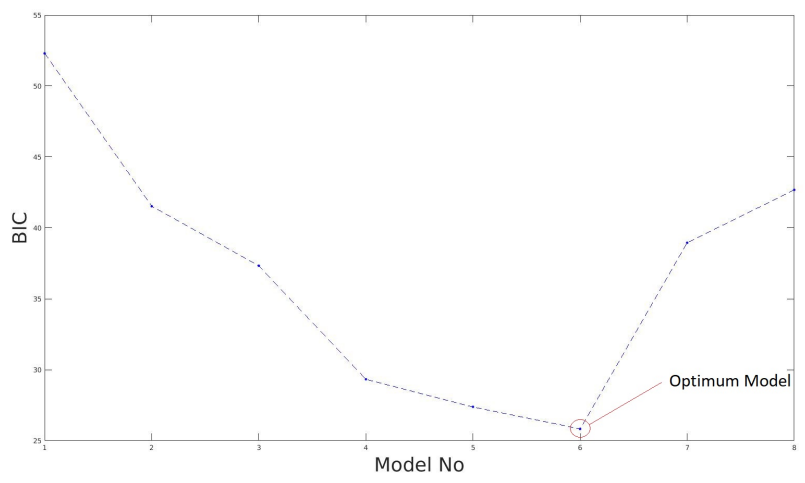

Figure 19: BIC for Different Models

The optimum model from the above chart corresponds to a reduced order model with six $\beta$ parameter values. Its discrepancy function takes the following form. 


$$
\delta=\sum_{i=1}^{5} \beta_{i, 1} \phi_{1}\left(x_{i}\right)+\left(\beta_{1,1,5,1}\right) \phi_{1}\left(x_{1}\right) \phi_{1}\left(x_{5}\right)
$$

Notice that only first order basis functions are required for the above model. It takes into account all main effects represented by the following term.

$$
\sum_{i=1}^{5} \beta_{i, 1} \phi_{1}\left(x_{i}\right)
$$

The second term incorporates only a single two-way interaction, corresponding to methane pressure and the inverse temperature.

$$
\left(\beta_{1,1,5,1}\right) \phi_{1}\left(x_{1}\right) \phi_{1}\left(x_{5}\right)
$$

The optimized model produces the following model prediction.
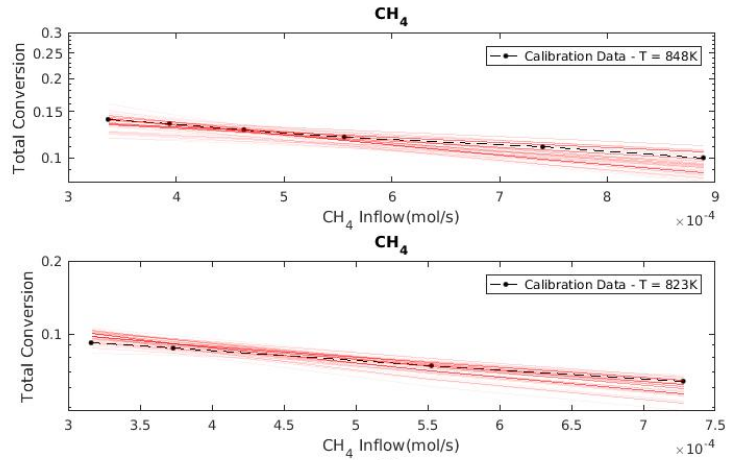

$\mathrm{CH}_{4}$
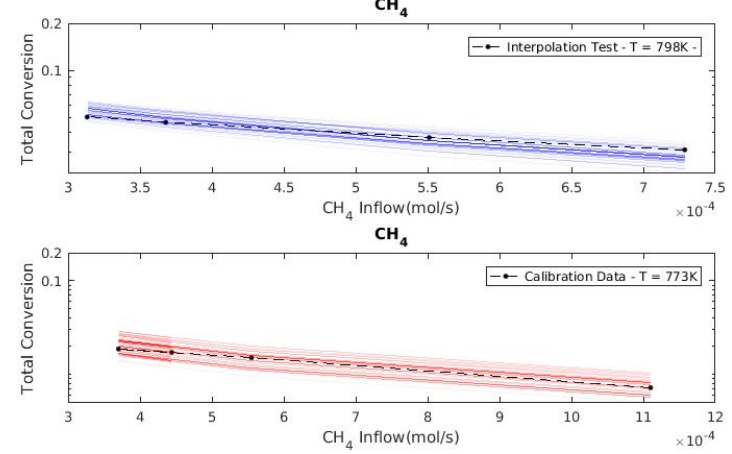

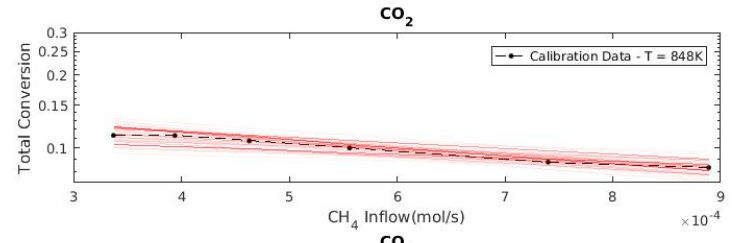

$\mathrm{CO}_{2}$

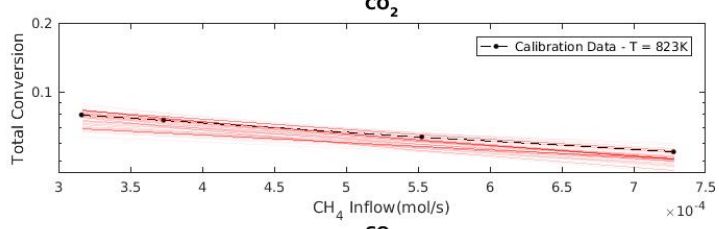

$\mathrm{CO}_{2}$

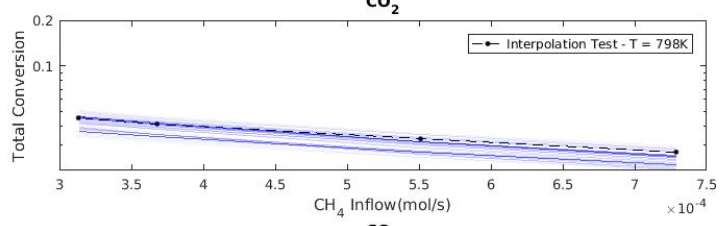

$\mathrm{CO}_{2}$

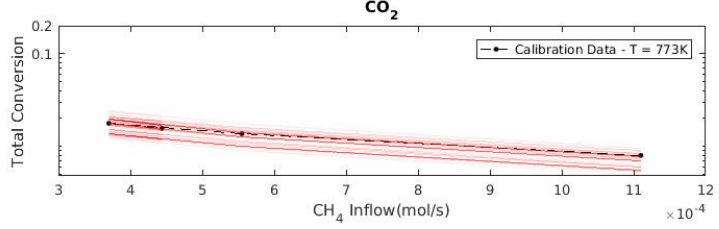

Figure 20: Optimized Model Prediction

The optimized model predictions show an acceptable amount of uncertainty with thin bands. A satisfactory coverage of experimental data is also observed. It also passes the test for interpolation, showing good coverage of the interpolation data. A slightly larger band is observed for interpolation data and the data corresponding to $\mathrm{T}=773 \mathrm{~K}$. A possible reason for this small difference could be that there were more 
data points in the calibration data for higher temperatures $(\mathrm{T}=848 \mathrm{~K}$ and $823 \mathrm{~K})$ than for $\mathrm{T}=773 \mathrm{~K}$.

\subsection{Plug Flow Reactor(PFR)}

So far the model in this work has only been concerned with lab-scale CSTR. Extending this model for process design will involve extrapolation to a much larger scale. A plug flow reactor model(PFR) is used for this purpose. The PFR is a model used to describe chemical reactions in continuous, flowing systems of cylindrical geometry. The PFR model is used to predict the behavior of chemical reactors of such design, so that key reactor variables, such as the dimensions of the reactor, can be estimated.

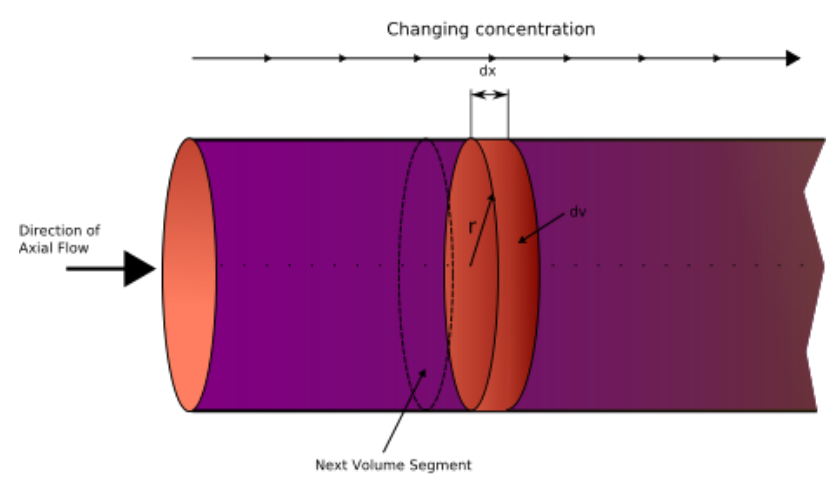

Figure 21: Plug Flow Reactor[44]

Fluid going through a PFR may be modeled as flowing through the reactor as a series of infinitely thin coherent "plugs", each with a uniform composition, traveling in the axial direction of the reactor, with each plug having a different composition from the ones before and after it. The key assumption is that as a plug flows through a PFR, the fluid is perfectly mixed in the radial direction but not in the axial direction (forwards or backwards). Each plug of differential volume is considered as a separate entity, effectively an infinitesimally small continuous stirred tank reactor(CSTR), limiting to infinitely small volume. The CSTRs can be assumed to be at steadystate. This means that the steady-state outflow of a CSTR is the inflow of the CSTR next to it and so on. Applying this methodology to the optimum model obtained with calibration, an industrial scale simulation of the transient response of the model is conducted. This produces the following sets of predictions. 


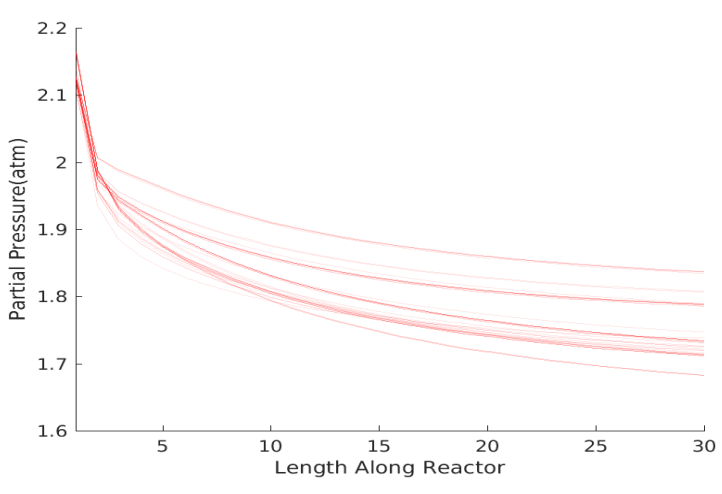

(a) $\mathrm{CH}_{4}$

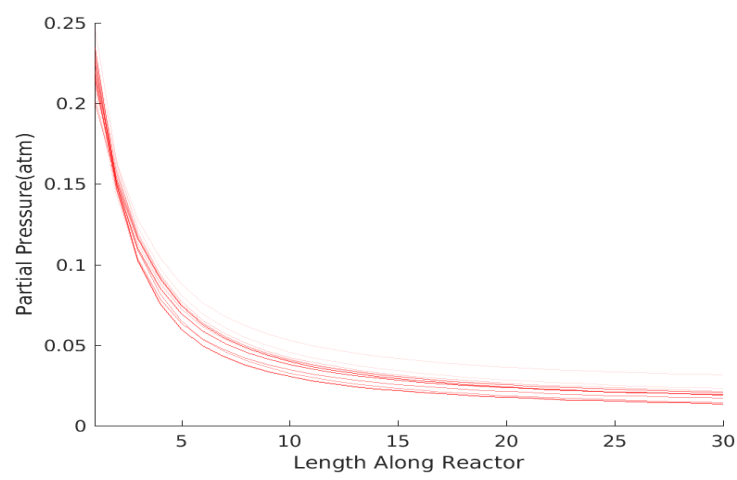

(c) $\mathrm{CO}_{2}$

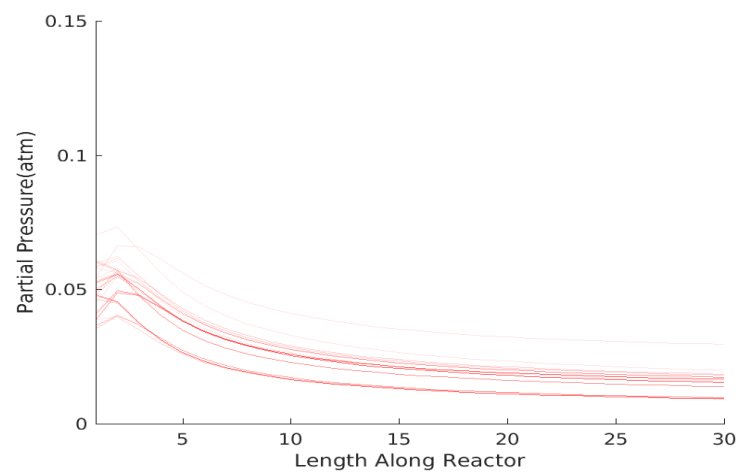

(e) $\mathrm{CO}$

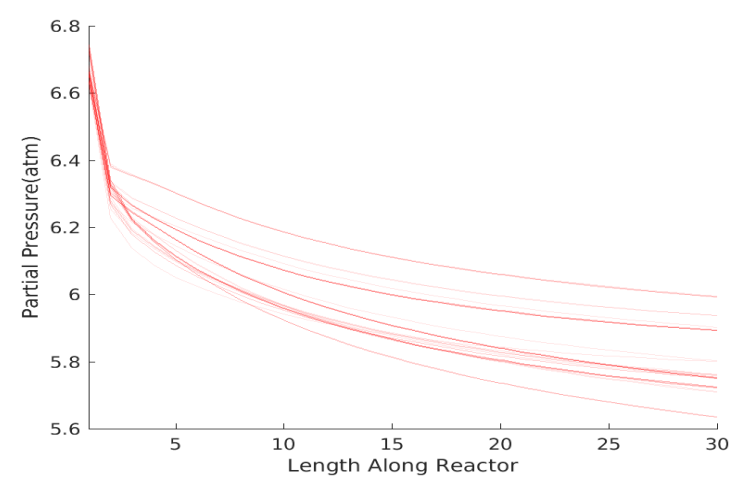

(b) $\mathrm{H}_{2} \mathrm{O}$

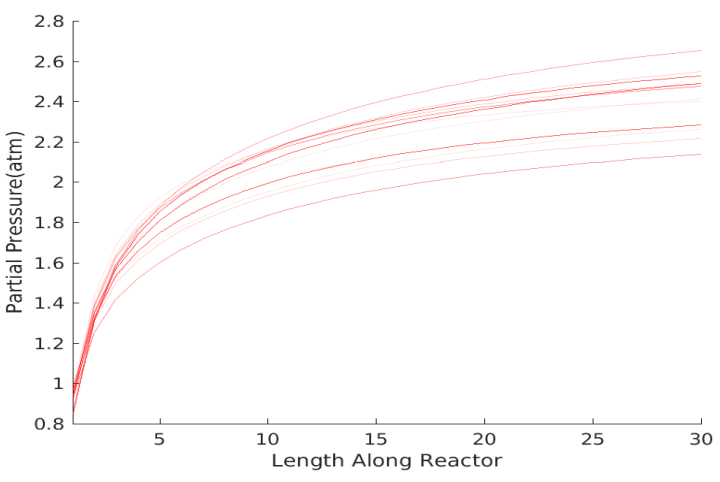

(d) $\mathrm{H}_{2}$

Figure 22: PFR Output With Optimum Model

As seen from figure, a band of predictions is obtained from the set of converged optimum models. As is the case with individual CSTRs, the thickness of the bands represents the uncertainty associated with the predictions. From the figure for $\mathrm{CH}_{4}$, $\mathrm{H}_{2} \mathrm{O}$ and $\mathrm{H}_{2}$, it can be seen that the bands get thicker the more the observations move along the length of the reactor. This is expected, since extrapolating the optimum model further and further along the length of the reactor will lead to increased uncertainty about the predictions.

Industrial scale data, generated from pending work, will be used to validate these predictions in subsequent work. 


\subsection{Discussions and Future Work}

Overall, the reduced model obtained in this work show good agreement with the experimental data present in literature. It also shows satisfactory interpolation capabilities, producing output matching experimental data for conditions not present in the calibration data. In short, this work proves the validity of the framework to create reduced models using dynamic discrepancy. Further work can also be conducted from the thread of this work.

From the perspective of the SMR process and process modeling, one of the limitations is that only the steady-state response of the model has been used for validation in this work. This is due to the lack of suitable experimental data pertaining to transient conditions in literature. Therefore, further work can involve obtaining/creating data for transient state responses(possibly from creating a high-fidelity model to generate data for varying conditions) and building/validating the reduced model from the obtained data. It would also include obtaining/creating suitable industrial scale data to validate the PFR predictions.

Finally from the viewpoint of the model reduction and dynamic discrepancy, a lot of manual effort had to be employed to arrive at the final optimum discrepancy function using a series of calibrations. This can be automated with a routine that builds the discrepancy terms by itself after each calibration, eventually building a complete reduced model from only available data and the reduced reaction network. It may even be feasible to automate the process of model reduction by creating an algorithm that can identify the relevant dependencies and omit the correct species. If both these objectives are achieved, then the entire process described in this work will be purely data driven model building, requiring very little human supervision. 


\section{Acknowledgements}

I would like to personally thank the following people for their help and support throughout this endeavor:

- Dr. David Mebane for serving as my advisor providing insight and encouragement.

- Dr. Fernando Lima for acting as a co-advisor and his research group for providing suggesions regarding the chemical engineering aspects of this project.

- Dr Sierros for being a part of my defense committee.

- My parents, MD Shafiqul Alam and Afroza Begum, for their love and support.

- My ESMS lab group for providing a supportive environment conducive to learning. 


\section{Citations}

[1] A Tour of The Top 10 Algorithms for Machine Learning Newbies, James Le, https://towardsdatascience.com/a-tour-of-the-top-10-algorithms-for-machine-learningnewbies-dde4edffae11

[2] Klaus R. Schneider - Thomas Wilhelm - Model reduction by extended quasisteady-state approximation, J. Math. Biol. 40, 443-450 (2000) Mathematical Biology, Digital Object Identifier (DOI):10.1007/s002850000026

[3] A.S. Tomlin, R.B. Brad, A systematic lumping approach for the reduction of comprehensive kinetic models, Proceedings of the Combustion Institute, Volume 30, Issue 1, 2005, Pages 1309-1316, ISSN 1540-7489, https://doi.org/10.1016/.. j.proci.2004.08.001. [4] SENSITIVITY ANALYSIS IN CHEMICAL KINETICS, H.Rabitz, M. Kramer, 1 and D. Daco - International Journal of Chemical Kinetics Volume 16, Issue 5 (https://doi.org/10.1002/kin.550160506)

[5] Balanced Model Reduction via Proper Orthogonal Decomposition - K. Willcox and J. Peraire, AIAA JOURNAL, Vol. 40, No. 11, November 2002

[6]Kennedy, M. C. and O'Hagan, A. (2001), Bayesian calibration of computer models. Journal of the Royal Statistical Society: Series B (Statistical Methodology), 63: 425464. doi:10.1111/1467-9868.00294

[7] Learning about physical Parameters: the importance of model discrepancy - Jenný Brynjarsdóttir and Anthony OHagan 2014 Inverse Problems 30114007

[8] V. Roshan Joseph Shreyes N. Melkote (2009) Statistical Adjustments to Engineering Models, Journal of Quality Technology, 41:4, 362-375

[9] Sargsyan, K. , Najm, H. N. and Ghanem, R. (2015), On the Statistical Calibration of Physical Models. Int. J. Chem. Kinet., 47: 246-276. doi:10.1002/kin.20906

[10] K. Sham Bhat, David S. Mebane, Priyadarshi Mahapatra Curtis B. Storlie (2017), Upscaling Uncertainty with Dynamic Discrepancy for a Multi-Scale Carbon Capture System, Journal of the American Statistical Association, 112:520, 1453-1467, DOI: $10.1080 / 01621459.2017 .1295863$

[11] Embedded Error Representation for Bayesian Model Calibration -Khachik Sargsyan, Xun Huan, Habib N. Najm https://arxiv.org/abs/1801.06768

[12] Yanyan He, Dongbin Xiu, Numerical strategy for model correction using physical constraints, Journal of Computational Physics, Volume 313, 2016, Pages 617-634, ISSN 0021-9991, https://doi.org/10.1016/j.jcp.2016.02.054.

[13] Combining Field Data and Computer Simulations for Calibration and Prediction, Dave Higdon, Marc Kennedy, James C. Cavendish, John A. Cafeo, and Robert D. Ryne, SIAM Journal on Scientific Computing 2004 26:2, 448-466

[14] Validating Predictions of Unobserved Quantities - Todd A. Oliver, Gabriel Terejanu, Christopher S. Simmons, Robert D. Moser, Validating predictions of unobserved quantities, Computer Methods in Applied Mechanics and Engineering, Volume 283, 2015, Pages 1310-1335, ISSN 0045-7825, https://doi.org/10.1016/j.cma.2014.08.023. 
[15] Jianguo Xu and Gilbert Froment. Methane steam reforming, methanation and water-gas shift: I intrinsic kinetics. AIChE Journal, 35(1):88-96, 1989.

[16] Aylin C, igdem Kone and Tayfun Buke. Forecasting of CO2 emissions from fuel combustion using trend analysis. Renewable and Sustainable Energy Reviews, 14(9):2906 - 2915, 2010.

[17] Esmail M. A. et. al. Mokheimer. On the Modeling of Steam Methane Reforming. Journal of Energy Resources Technology, 137:12001-1 - 12001-11, 2015.

[18] John N. Armor. The multiple roles for catalysis in the production of H2. Applied Catalysis A: General, 176(2):159 - 176, 1999.

[19] Pegah Ghanbari Bavarsad. Energy and exergy analysis of internal reforming solid oxide fuel cell-gas turbine hybrid system. International Journal of Hydrogen Energy, 32(17):4591 - 4599, 2007.

[20] Bousquet, P., S. C. Tyler, P. Peylin, G. R. Van Der Werf, C. Prigent, D. A. Hauglustaine, E. J. Dlugokencky, J. B. Miller, P. Ciais, J. White, L. P. Steele, M. Schmidt, M. Ramonet, F. Papa, J. Lathière, R. L. Langenfelds, C. Carouge, and E.-G. Brunke. "Contribution of anthropogenic and natural sources to atmospheric methane variability." Nature 443, no. 7110 (2006): 439-443.

[21] K.Spokasa, J.Bognerb, J.P.Chantonc, M.Morcetd, C.Arand, C.Graffa,Y. MoreauLeGolvane, I.Hebef, Methane mass balance at three landfill sites: What is the efficiency of capture by gas collection systems? WASTE MANAGEMENT Volume: 26, Issue: 5,Pages: 516-525

[22] J. K. Rajesh, Santosh K. Gupta, G. P. Rangaiah, and Ajay K. Ray. Multiobjective optimization of steam reformer performance using genetic algorithm. Industrial Engineering Chemistry Research, 39(3):706-717, 2000.

[23] YuzhangWang, Fumihiko Yoshiba, Makoto Kawase, and TakaoWatanabe. Performance and effective kinetic models of methane steam reforming over ni/ysz anode of planar SOFC. International Journal of Hydrogen Energy, 34(9):3885 - 3893, 2009. 52

[24] Anna Sciazko, Yosuke Komatsu, Grzegorz Brus, Shinji Kimijima, and Janusz S. Szmyd. A novel approach to the experimental study on methane/steam reforming kinetics using the orthogonal least squares method. Journal of Power Sources, 262(0):245 - 254, 2014.

[25] W. Sangtongkitcharoen, S. Assabumrungrat, V. Pavarajarn, N. Laosiripojana, and P. Praserthdam. Comparison of carbon formation boundary in different modes of solid oxide fuel cells fueled by methane. Journal of Power Sources, 142(1-2):75 80,2005 .

[26] Model Reduction for Chemical Kinetics: An Optimization Approach. Linda Petzold Dept. of Mechanical and Environmental Engineering, University of California, Santa Barbara, CA 93106 Wenjie Zhu Dept. of Computer Science, University of Minnesota, Minneapolis, MN 55455

[27] A.M. Aitani. Processes to enhance refinery-hydrogen production. International Journal of Hydrogen Energy, 21(4):267 - 271, 1996. 53

[28] Y Ding and E Alpay. Adsorption-enhanced steam-methane reforming. Chemical 
Engineering Science, 55(18):3929 - 3940, 2000.

[29] Kaihu Hou and Ronald Hughes. The kinetics of methane steam reforming over a ni/_al2ocatalyst.ChemicalEngineering Journal, 82(1-3) : 311-328, 2001.

[30] James Van Hook. Methane-steam reforming. Catalysis Review. Science and engineering, 21(1):1 - 51, 1980.

[31] W. W. Akers and D. P. Camp. Kinetics of the methane-steam reaction. AIChE Journal, 1(4):471-475, 1955.

[32] J. R. H. Ross and M. C. F. Steel. Mechanism of the steam reforming of methane over a coprecipitated nickel-alumina catalyst. Chemical Society Faraday Transacitons I, 69:11 - 22, 1973.

[33] P. Munster and H.J. Grabke. Kinetics of the steam reforming of methane with iron, nickel, and iron-nickel alloys as catalysts. Journal of Catalysis, 72(2):279 - 287, 1981.

[34] Jianguo Xu and Gilbert Froment. Methane steam reforming: Ii. diffusional limitations and reactor simulation. AIChE Journal, 35(1):97 - 103, 1989.

[35] M. A. Soliman, A. M. Adris, A. S. Al-Ubaid, and S. S. E. H. El-Nashaie. Intrinsic kinetics of nickle/calcium aluminate catalyst for methane steam reforming. Journal of Chemical Technology and Biotechnology, 55(2):131-138, 1992.

[36] Multi-objective Optimization of Steam Reformer Performance Using Genetic Algorithm J. K. Rajesh,Santosh K. Gupta,†, G. P. Rangaiah, and, and Ajay K. Ray* Industrial Engineering Chemistry Research 200039 (3), 706-717

DOI: $10.1021 /$ ie9905409

[37] J. C. De Deken, E. F. Devos, and F. Froment. Steam reforming of natural gas intrinsic kinetics, diffusional influences, and reactor design. ACS Sympoesium Series, 196:181- 197, 1982.

[38] Evan Ford,MSc Thesis - A Bayesian Approach to Reduced Order Modeling in Catalytic Steam Reforming, Advisor - David. S Mebane.

[39] Wikipedia: Continuously Stirred Tank Reactor

[40] Yanyan He, Dongbin Xiu, Numerical strategy for model correction using physical constraints, Journal of Computational Physics, Volume 313,2016,Pages 617-634,ISSN 0021-9991, https://doi.org/10.1016/j.jcp.2016.02.054.

[41]Brian J. Reich, Curtis B. Storlie Howard D. Bondell (2009) Variable Selection in Bayesian Smoothing Spline ANOVA Models: Application to Deterministic Computer Codes, Technometrics, 51:2, 110-120, DOI: 10.1198/TECH.2009.0013

[42] Curtis B. Storlie, William A. Lane, Emily M. Ryan, James R. Gattiker David M. Higdon (2015) Calibration of Computational Models With Categorical Parameters and Correlated Outputs via Bayesian Smoothing Spline ANOVA, Journal of the American Statistical Association, 110:509, 68-82, DOI: 10.1080/01621459.2014.979993

[43] https://www.mathworks.com/matlabcentral/fileexchange/7173-grabit

[44] Wikipedia: Plug Flow Reactor Model 